Award Number: W81XWH-04-1-0827

TITLE: Potentiation of Prostate Cancer Radiotherapy Using Antiangiogenic and Antitumor Therapies

PRINCIPAL INVESTIGATOR: Bruce M. Fenton, Ph.D.

CONTRACTING ORGANIZATION: University of Rochester

Rochester, NY 14642

REPORT DATE: October 2007

TYPE OF REPORT: Final

PREPARED FOR: U.S. Army Medical Research and Materiel Command Fort Detrick, Maryland 21702-5012

DISTRIBUTION STATEMENT: Approved for Public Release;

Distribution Unlimited

The views, opinions and/or findings contained in this report are those of the author(s) and should not be construed as an official Department of the Army position, policy or decision unless so designated by other documentation. 


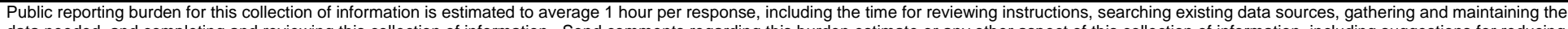

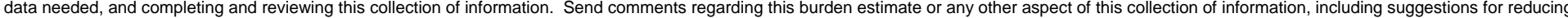

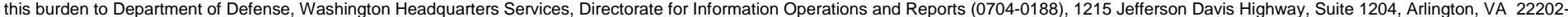

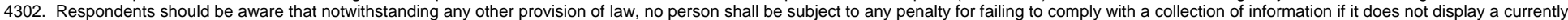
valid OMB control number. PLEASE DO NOT RETURN YOUR FORM TO THE ABOVE ADDRESS.

\begin{tabular}{l|l|l|} 
1. REPORT DATE (DD-MM-YYYY) & 2. REPORT TYPE & 3. DATES COVERED (FrOm - To)
\end{tabular}

01-10-2007

\section{TITLE AND SUBTITLE}

10 SEP 2004 - 9 SEP 2007

Potentiation of Prostate Cancer Radiotherapy Using Antiangiogenic and Antitumor Therapies

5a. CONTRACT NUMBER

5b. GRANT NUMBER

W81XWH-04-1-0827

5c. PROGRAM ELEMENT NUMBER

6. AUTHOR(S)

Bruce M. Fenton, Ph.D.

5d. PROJECT NUMBER

5e. TASK NUMBER

E-Mail: bruce.fenton@rochester.edu

5f. WORK UNIT NUMBER

7. PERFORMING ORGANIZATION NAME(S) AND ADDRESS(ES)

8. PERFORMING ORGANIZATION REPORT NUMBER

University of Rochester

Rochester, NY 14642

9. SPONSORING I MONITORING AGENCY NAME(S) AND ADDRESS(ES)

10. SPONSOR/MONITOR'S ACRONYM(S)

U.S. Army Medical Research and Materiel Command

Fort Detrick, Maryland 21702-5012

11. SPONSOR/MONITOR'S REPORT NUMBER(S)

12. DISTRIBUTION / AVAILABILITY STATEMENT

Approved for Public Release; Distribution Unlimited

\section{SUPPLEMENTARY NOTES}

\section{ABSTRACT}

The focus of this grant was to gauge the pathophysiological effects of combinations of radiotherapy and a variety of antiangiogenic agents by studying corresponding changes in vascular maturity and function in PC-3 and DU145 human prostate xenografts. Perfusion, apoptosis, proliferation, and hypoxia indices as well as pericyte and basement membrane coverage were quantified using image analysis of immunohistochemically stained frozen tumor sections. Results argue against a treatment-induced functional normalization of the tumor vasculature following combination therapy. Rather than tightening pericytes, combination treatments loosened pericyte-vessel and pericytebasement membrane associations. Despite reductions in oxygenation and vessel densities, tumor progression was minimal during extended combination treatment, most likely due to continued vascular destruction and the prevention of new vessel growth. Surprisingly, alternative scheduling of antiangiogenic drugs and radiation had minimal effects on tumor progression or pathophysiology.

\section{SUBJECT TERMS}

Angiogenesis, tumor vasculature, hypoxia, tumor pathophysiology

\section{SECURITY CLASSIFICATION OF:}

\begin{tabular}{c|c|c}
\hline a. REPORT & b. ABSTRACT & c. THIS PAGE \\
$U$ & $U$ & $U$
\end{tabular}

17. LIMITATION OF ABSTRACT

UU
18. NUMBER OF PAGES

25 19a. NAME OF RESPONSIBLE PERSON USAMRMC

19b. TELEPHONE NUMBER (include area code) 


\section{Table of Contents}

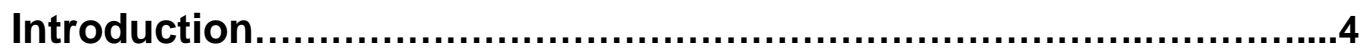

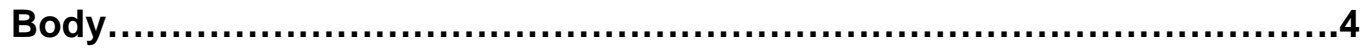

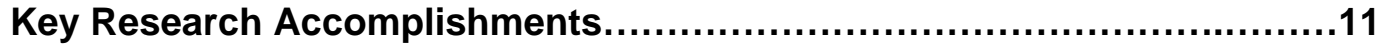

Reportable Outcomes...............................................................

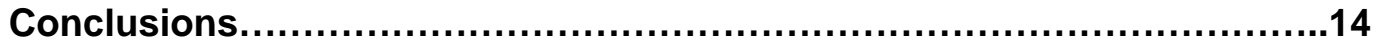

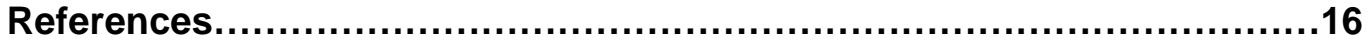

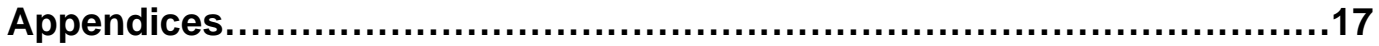




\section{Introduction:}

Although antiangiogenic strategies have been shown highly promising in preclinical studies and some recent phase II and III clinical trials, generally only combinations of antiangiogenic and cytotoxic therapies have shown clinical effectiveness. An ongoing question regarding such combined therapies has been whether conventional therapies such as radiotherapy (RT) are enhanced or compromised by antiangiogenic agents. Although antiangiogenic strategies might be expected to destroy tumor vasculature and thereby deprive the tumor of radiosensitizing oxygen, results are inconclusive. Some workers have found decreased tumor oxygenation and blood flow following such agents, while others have shown the opposite. Concurrent, post-RT, and pre-RT combination scheduling with antiangiogenics have all been shown advantageous. The past three years' studies were designed to evaluate the consequences of combining fractionated radiation with various antiangiogenic strategies, as well as to investigate the ramifications of alternative scheduling of therapies. Key questions were whether vascular density decreases following 1-3 wks of single or combination treatment, and if so, does tumor hypoxia increase, or instead decrease in line with vascular normalization? Given the well-known dependence of radiosensitivity on tumor oxygen levels, any increase in hypoxia would presumably also compromise further combination therapy. A second objective was to compare alterations in the tightness and coverage of pericyte markers and basement membrane, to determine the relation between pericyte coverage, selective vascular ablation, and the retention of "vascular ghosts" following therapy.

\section{Body:}

Over the three years of this project, we have carried out a progression of studies, all designed to better understand the pathophysiological consequences of combining RT with antiangiogenic therapy. Year 1 concentrated on establishing the prostate xenograft tumor models and measuring tumor response to both fractionated RT and either DC101 (anti-VEGFR-2) or A12 (anti-IGF-1R) from ImClone. Years 2 and 3 progressed to new drugs from Pfizer Global Research, including AG-028262 (a receptor tyrosine kinase inhibitor of VEGFR-1,2,3) and AG-013736 (an inhibitor of VEGFR-1,2,3, PDGFR $\beta$, and c-kit). Our goal in administering the fractionated RT was to determine a dose that produced some slowing of tumor growth without total control, such that later synergistic or additive effects of the antiangiogenics would be readily apparent. For this purpose, daily fractions of $2 \mathrm{~Gy}$, which are similar to clinical scheduling of RT fractions, were found to be appropriate for both the PC-3 and DU145 tumor models.

Response to A12, DC101, RT, and combination therapies in DU145 xenografts: Our first tumor response experiments investigated the effects of fractionated RT, DC101, and A12 alone on the growth of DU145 tumors. All treatments significantly delayed tumor growth, A12 producing the least effect and DC101 the most striking. The addition of the A12 to either RT or DC101 had no substantial 
added effect on tumor growth, and we subsequently discontinued experiments with this agent. We also ceased DC101 studies at this time, since the newly available Pfizer drugs appeared more promising with less toxicity.

Response to AG-028262, RT, and combination therapy in DU145 xenografts: We next determined the dose dependence of AG-028262. To delay tumor growth without complete inhibition, suboptimal doses of $40 \mathrm{mg} / \mathrm{kg}$ were found appropriate. As an initial proof-of-principle experiment, AG-028262 was combined with RT using three alternative schedules: 1) 1 wk RT followed by 1 wk with a combination of AG-028262 + RT, 2) 1 wk AG-028262 followed by 1 wk combination, or 3) 2 wks of the combination RT + AG-028262. Single agent RT or AG-028262 were also included for comparison. At the end of $2 \mathrm{wks}$, the 2-wk combination therapy resulted in a slight decrease in tumor volume with respect to the other 2 schedules, but the effect was short-lived and tumors from all three schedules progressed to similar volumes by the end of week five. This similarity in response among the three treatment schedules was somewhat unexpected in light of the anticipated decrease in tumor oxygenation following the antiangiogenic drug. Assuming the drug decreased oxygenation, RT would have been expected to be less effective. The next series of experiments were designed to characterize changes in tumor oxygenation and vascular configuration following treatment.

Image analysis of pathophysiological indices: Image analysis algorithms to quantitate tumor pathophysiological changes were developed during year 2 of the grant. Tumor blood vessel spacing was determined using a combination of image segmentation and distance map filtering to obtain the distribution of distances to the nearest vessel. These distances (related to tumor blood vessel spacing) are reflective of the median distances over which oxygen and nutrients must diffuse to reach all cells of the tumor. Colocalized and thresholded images of panendothelial cell antigen staining, DiOC 7 (for perfusion), and TUNEL staining (for apoptosis) were obtained, and population densities were determined for each as well as for the overlap between TUNEL and perfused vessel staining (using custom ImagePro macros to obtain population densities within defined areas of interest). Percentage apoptotic vessels was calculated by dividing the overlap population density (which represents apoptotic endothelial cells) by vessel population density. Ki-67 stained images (proliferation marker) were quantified by determining \% positively stained area (again using ImagePro thresholding and counting operations). We acquired colocalized and thresholded stained images of panendothelial cell antigen and three pericyte markers: 1) PDGFR $\beta$ + cells, 2) NG2+ cells, and 3) $\alpha$-sma+ cells. Percent areas of each as well as the \% area of the overlap between endothelial and pericyte markers were determined (using ImagePro measurement operations within defined areas of interest).

Tumor response to AG-013736, RT, and combination treatment in DU145 and $P C-3$ xenografts: Unlike AG-028262, which targets only the VEGF receptors, and 
was thus expected to be primarily antiangiogenic, AG-013736 targets both VEGF and PDGF receptors, and was predicted to act as both an antiangiogenic and an antivascular agent due to the interdependence between PDGFRs and vascular pericyte coverage. Somewhat contradictory reports in the literature have shown that VEGFR-2 inhibition can either preferentially target and destroy non-pericyte coated blood vessels, or instead tighten pericyte-endothelial coverage, thus conferring a more mature phenotype on the vessels. A primary goal during years 2-3 was to specifically investigate these issues.

The culmination of our most significant studies on the response of DU145 tumors to combination therapies (over years 2-3) was published in Cancer Research this past summer ${ }^{1}$ (included in the Appendix) and will therefore only be briefly summarized here. In these experiments, dose response studies on the AG013736 were first conducted to determine a suboptimal dose for later combination studies with RT. Mice were then treated for 1-3 wks with either: 1) AG-013736 alone, 2) fractionated RT, or 3) the combination. Tumor growth inhibition was similar for either the RT or AG-013736 monotherapies, but markedly increased for the combination. To better understand the accompanying pathophysiological changes, total and perfused blood vessel spacing and hypoxia were next quantified from frozen tumor sections. All of the treatments significantly increased total and perfused blood vessel spacing, but only AG013736 and the combination produced an increase in tumor hypoxia. Because alterations in total and perfused vessel spacings were generally proportionate, functional vasculature was likely not selectively targeted by the combination therapy. With the combination, however, hypoxia progressively increased with each succeeding wk of therapy. Based on TUNEL staining, neither overall tumor cell apoptosis nor endothelial cell apoptosis was significantly increased by any of the treatments, although perfused vessel endothelial apoptosis was significantly higher for the combination. Accompanying the increase in apoptosis, tumor cell proliferation decreased at both wks 2 and 3 for the combination.

We next studied alterations in pericyte coverage with treatment, using PDGFR $\beta$ as a marker of pericyte progenitor cells, and $\alpha$-sma as a marker of a more mature pericyte phenotype. Previous studies have suggested that VEGFR-2 inhibitors alone can lead to a "vascular normalization", defined as a combination of: 1) selective pruning of excess tumor vessels, 2) an increase in pericyte coverage, and 3) a transient improvement in tumor oxygenation. This contrasted with our findings, in which perfused and nonperfused vessels were nonselectively ablated by the combination treatments. We observed an initial increase in $\alpha$-sma coverage, combined with increased dissociation of both $\alpha$-sma+ and PDGFR $\beta+$ pericytes. This supports a selective ablation of non- $\alpha$-sma+ coated vessels, rather than a recruitment of $\alpha$-sma+ pericytes. Following extended combination therapy, both $\alpha$-sma+ and PDGFR $\beta+$ pericytes were significantly dissociated from the blood vessels. In addition, basement membrane sleeves (type IV collagen+) separated from the endothelial layer of the blood vessels. Together, these data suggest that the net effect of the combination treatment is to 
selectively destroy endothelial cells, while at the same time stripping pericytes from their associated basement membranes.

In summary, these findings argue against a treatment-induced functional normalization of the tumor vasculature when applying combination therapy. Rather than tightening pericytes, AG-013736 and the combination treatment served instead to loosen pericyte-vessel and pericyte-basement membrane associations in our tumor models. Treatment substantially reduced total and functional vascular densities, but overall tumor hypoxia progressively increased, in contrast to the normalization hypothesis. Despite the reduction in oxygenation, tumor progression was minimal over three weeks of combination treatment, most likely due to continued vascular destruction and the prevention of new vessel growth.

\section{Alternative scheduling of combination treatment in PC-3 and DU145 xenografts:}

In the previous experimental design, combination therapy was scheduled such that AG-013736 was delivered following RT, under the assumption that pretreatment with the antiangiogenic agent would reduce tumor perfusion and thereby compromise RT. In light of more accurate information regarding the expected pharmacodynamics of AG-013736 treatment, and in view of the expected potentiation of RT effects by AG-013736, our combination treatment scheduling for the PC-3 tumors was modified in comparison to the previous DU145 studies. Rather than administering the AG-013736 immediately following each fraction of radiation, two schedules were contrasted, i.e., the drug was administered either $1 \mathrm{hr}$ pre- or $1 \mathrm{hr}$ post-irradiation.

Figure 1 summarizes the tumor growth curves for the various schedules. Fig. 1A is for DU145 tumors, while Figs. 1B,C, and D are for PC-3. Prior results ${ }^{1}$ demonstrated that combination therapy or AG-013736 alone resulted in progressively increasing tumor hypoxia, which would correspond to a decrease in overall radiosensitivity. In addition, based on previous in vitro results, it was expected that AG-013736 would sensitize endothelial cells to RT. Surprisingly, administering the drug $1 \mathrm{hr}$ pre-RT was no different than $1 \mathrm{hr}$ post-RT in terms of DU145 tumor growth rate (Fig. 1A).

Results were similar for the PC-3. Fig. 1B shows that the combination therapy is significantly better than either monotherapy, even when the tumors were as large as $560 \mathrm{~mm}^{3}$ at the initiation of treatment. Untreated tumors were frozen at a variety of different tumor volumes, and vessel spacing was contrasted to those for combination and monotherapies (Fig. 2). In the untreated tumors (UN), both total (black filled circles) and perfused (red filled circles) vessel spacings increased progressively with tumor growth, suggesting that the tumor cells are either outgrowing or destroying their own vasculature. At matched tumor volumes, both the monotherapies and the combination resulted in substantial increases in both total and perfused spacing (open symbols). For the combination treatment, in particular, tumor volume at day 7 remained unchanged from day 1 . Since vessel spacing at this time was significantly higher for both 

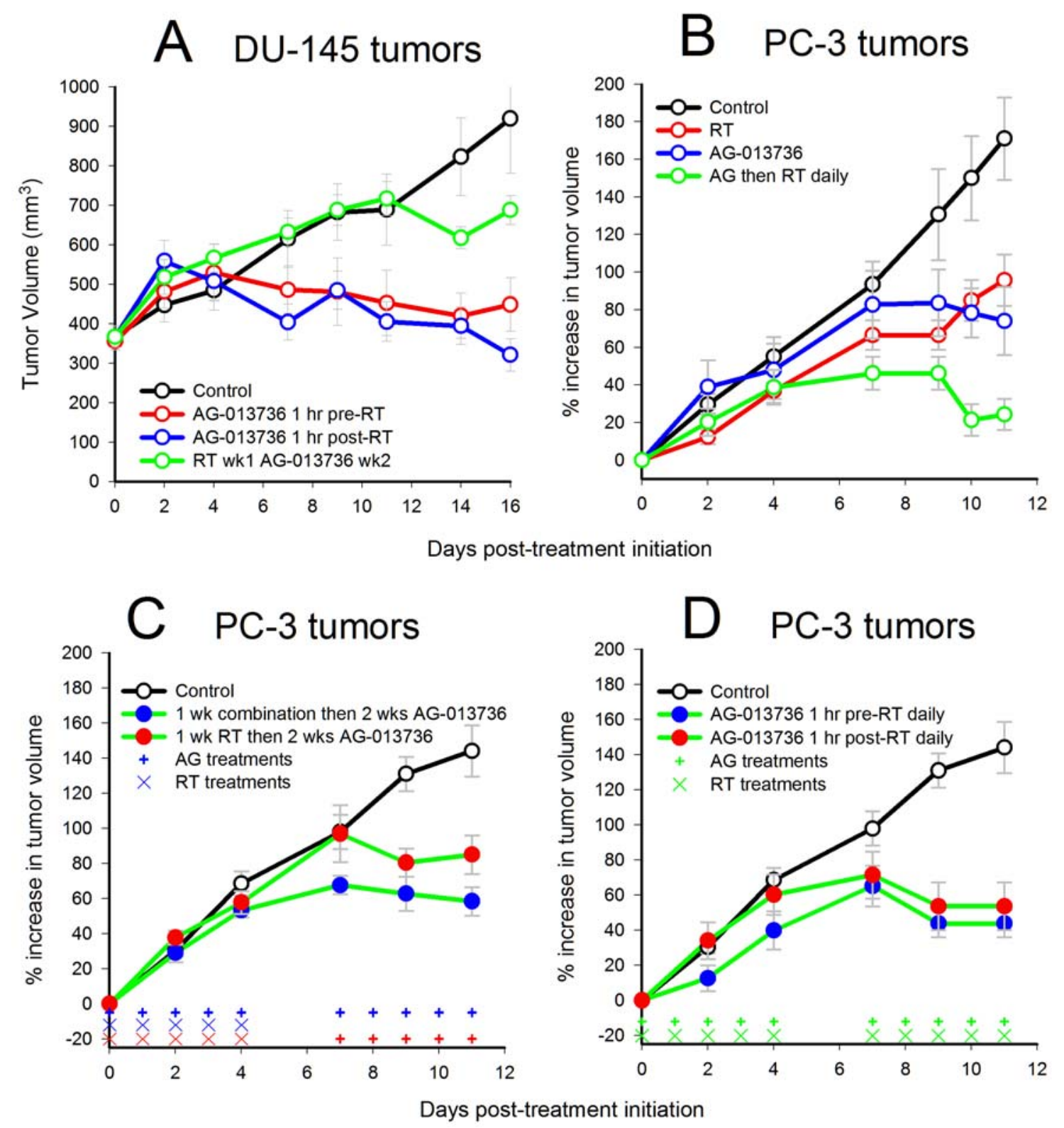

Figure 1 - Effect of various treatment schedules on growth of DU-145 and PC-3 tumors. In panels $C$ and $D$, treatment days are designated by the bottom row of symbols (+ for AG013736 and $X$ for RT)

total and perfused vessels, vessels must have been progressively eliminated during therapy, rather than the tumor cells outgrowing their vasculature as in the control tumors.

Once again the specific scheduling of RT and AG-013736 had no significant effect on tumor progression (Fig. 1D). In this particular experiment, total and perfused vessel spacing were also quantified (Fig. 3). In addition to small and large controls (un300 $=300 \mathrm{~mm}^{3}$ control; un600 $=600 \mathrm{~mm}^{3}$ control), tumors were frozen for AG-013736 or RT alone at day 7 (AG-d7, RT-d7), as well as for the AG-013736 pre-RT (AG-RT) and post-RT (RT-AG) combinations at days 4 and 7. In line with the growth curves, vascular spacing was similar following each of the alternative schedules. Tumor hypoxia was also quantified using EF5 uptake, but once again scheduling had no significant effect (data not shown). 


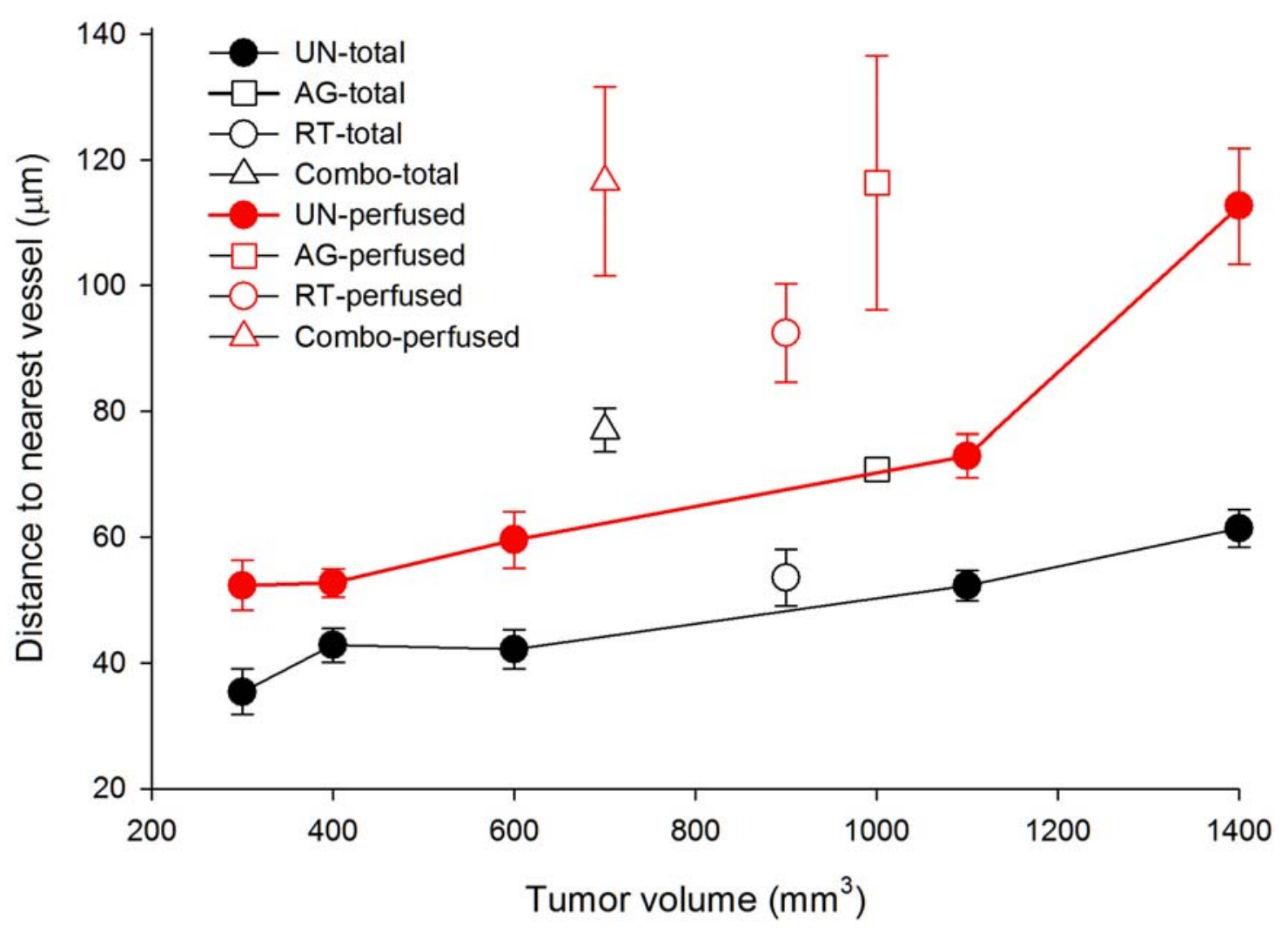

Figure 2 - Changes in total and perfused vessel spacing with increasing tumor volume in PC-3 tumors (filled symbols are untreated, AG = AG-013736, RT = 2 Gy fractionated radiation, combo = daily $R T+A G)$

Fig. 1C contrasts a schedule of either 1 wk RT or 1 wk of the combination, each followed by 2 wks of AG-013736 alone. In terms of overall growth rate, the alternative schedules were again very similar. For the combination treatment, tumors were frozen at six timepoints to further monitor temporal vascular response. Fig. 4 illustrates changes in total (open triangles) and perfused (open circles) vessel spacing after combination treatment. Treatment times are indicated by the "X" (for RT) and "+" (for AG-013736) symbols below the curves. The most striking changes were in perfused vessel spacing, which had already increased by day 2 post-treatment. From days 0-4, tumor volume increased, but with little vascular growth, resulting in a net decrease in the number of vessels (e.g., increased vessel spacing). By the end of treatment (day 18), however, functional vessels were actually being eliminated. With the alternate schedule of 1 wk RT followed by 2 wk AG-013736, both tumor volume (Fig. 1C) and vessel spacing (solid triangles in Fig. 4) were very similar to those starting with 1 wk of the combination treatment.

Variations in tumor and endothelial cell apoptosis, as well as proliferation, were also followed for these tumors. As shown in Fig. 5, neither total and perfused vessel endothelial cells showed any substantial changes in apoptosis during treatment, although overall tumor cell apoptosis did increase significantly by day 7. Neither overall tumor cell nor perivascular proliferation (Ki-67 staining) was altered at any timepoints (data not shown). In addition, tumor hypoxia showed no significant changes (data not shown). 


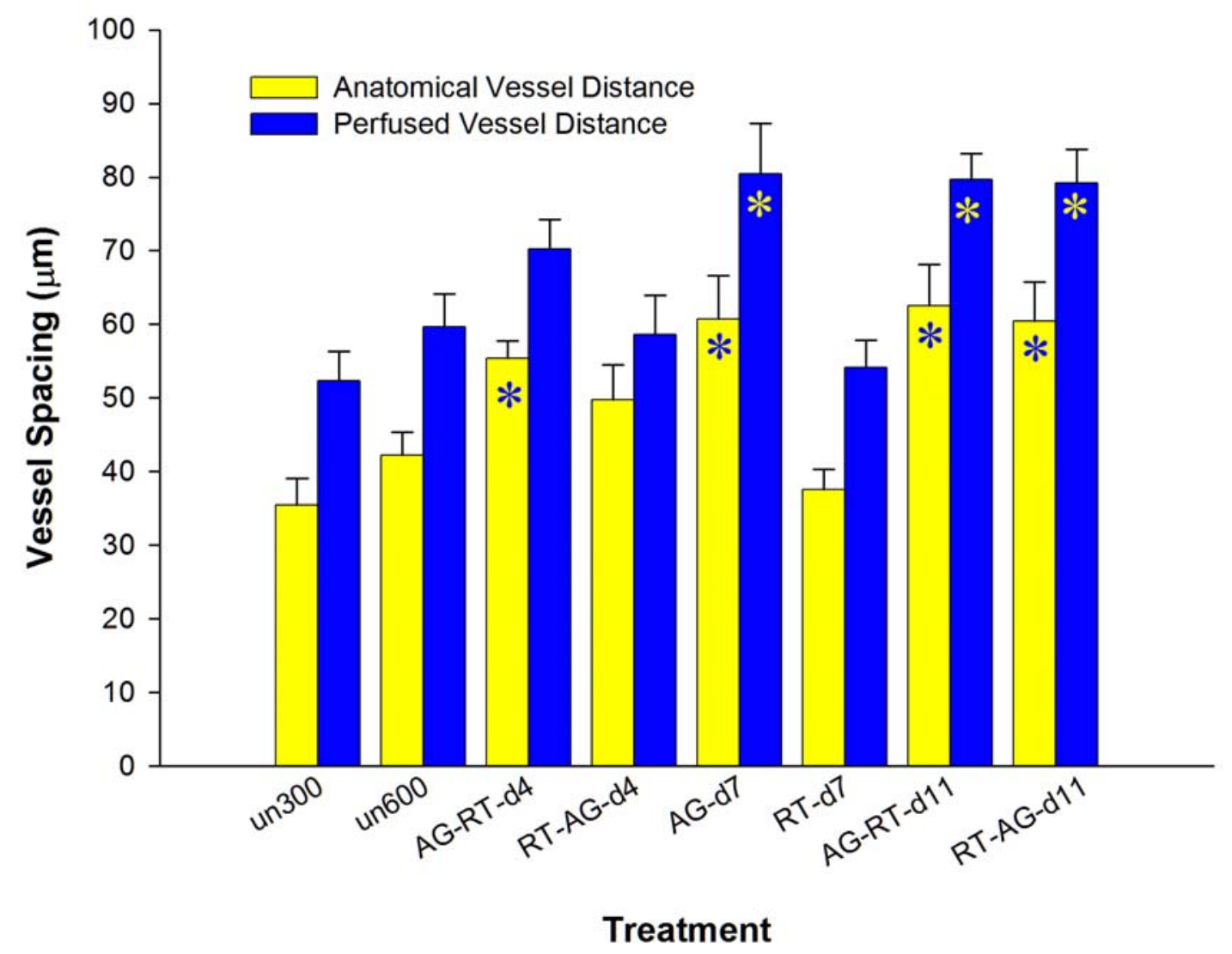

Figure 3 - Effect of single and combined treatments on total and perfused vessel spacing in PC-3 tumors. UN300 $=300 \mathrm{~mm}^{3}$ controls, UN600 $=600 \mathrm{~mm}^{3}$ controls, AG-RT-d4 = AG013736 administered $1 \mathrm{hr}$ pre-RT daily and frozen at day 4, RT-AG-d4 = AG-013736 administered $1 \mathrm{hr}$ post-RT daily and frozen at day 4.

Although AG-013736 was initially chosen in light of its combination inhibition of both VEGFRs and PDGFRs, PDGFR inhibition was minimal at clinically relevant doses ultimately chosen $(25 \mathrm{mg} / \mathrm{kg})$. Further studies are continuing using CP263,451 (a more specific inhibitor of PDGFR $\beta$ ) and CE-245,677 (a Tie-2 inhibitor), both also from Pfizer. 


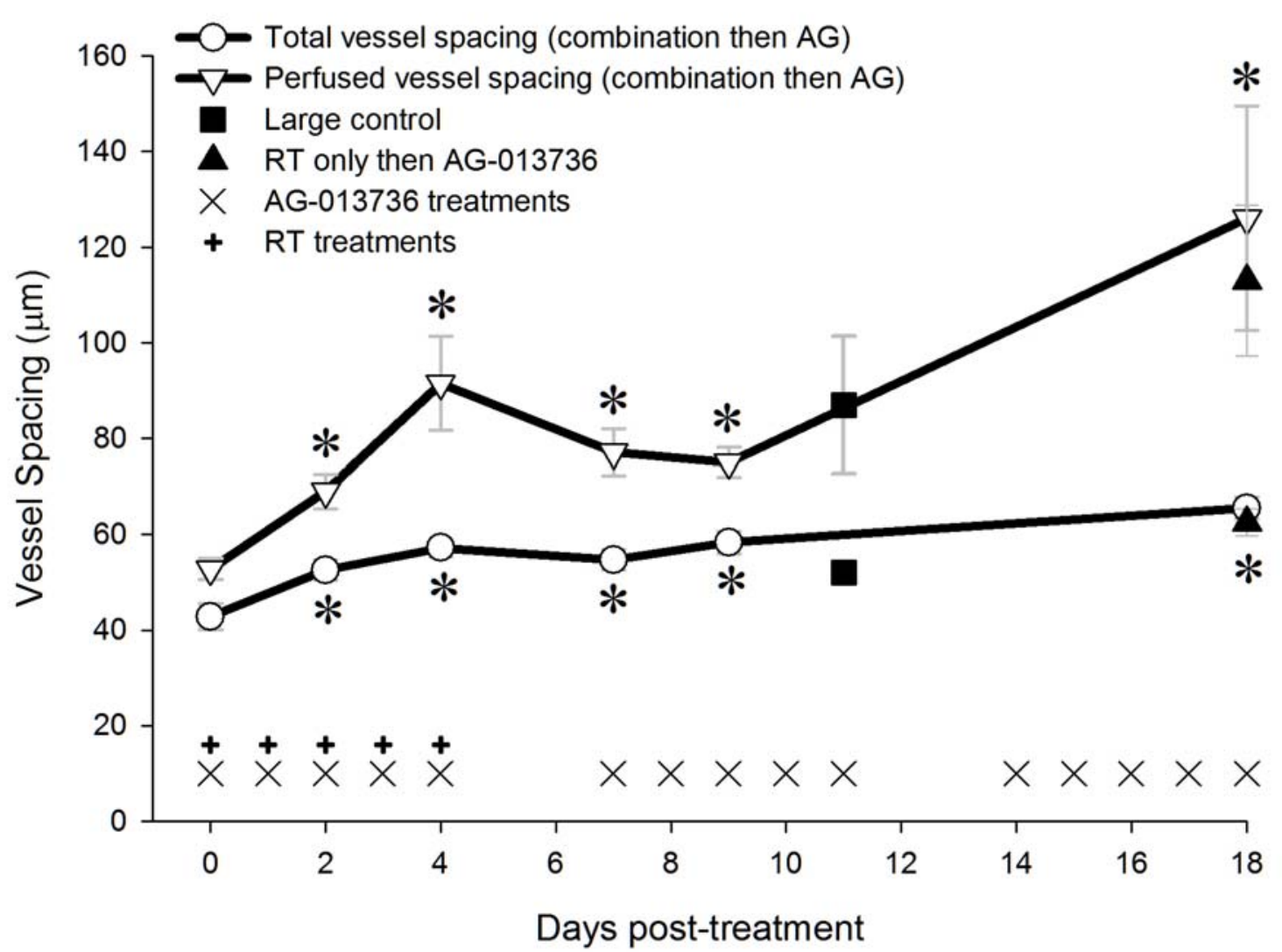

Figure 4 - Temporal changes in total and perfused vessel spacing in response to varied treatment scheduling in PC-3 tumors. Open symbols are combination treated for $1 \mathrm{wk}$ followed by AG- 013736 alone for 2 wks; filled squares are untreated; filled triangles are 2 Gy RT daily for 1 wk followed by AG-013736 daily for 2 wks. See text for further details.

\section{Key Research Accomplishments:}

1) Immunohistochemistry and image processing algorithms were optimized for pericyte and basement membrane markers (PDGFR- $\beta$, NG2, desmin, $\alpha$-sma, and type IV collagen), and automated ImagePro macros were developed to acquire composite images of overlap with total or perfused blood vessels. This provided measures of $\%$ coverage, $\%$ dissociation, and overall \% area for each.

2) In contrast to previous studies on response to antiangiogenic monotherapies, combinations of antiangiogenic agents with fractionated radiotherapy did not result in a "normalization" of tumor vasculature.

3) Rather than tightening pericytes, AG-013736 and the combination treatment served instead to loosen pericyte-vessel and pericyte-basement membrane associations in our tumor models.

4) Results support a selective ablation of non- $\alpha$-sma+ coated vessels, rather than a recruitment of $\alpha$-sma+ pericytes.

5) Combination treatment substantially reduced total and functional vascular densities by selectively destroying endothelial cells, while at the same time stripping pericytes from their associated basement membranes.

6) Despite an increase in overall tumor hypoxia, tumor response to combination therapy was striking, most likely due to continued vascular 
destruction and the prevention of new vessel growth for up to three weeks of treatment.

7) Contrary to expectations, the scheduling of antiangiogenic agents, i.e., pre- versus post-radiotherapy resulted in similar effects on tumor inhibition, vascular spacing, hypoxia, and pericyte coverage.

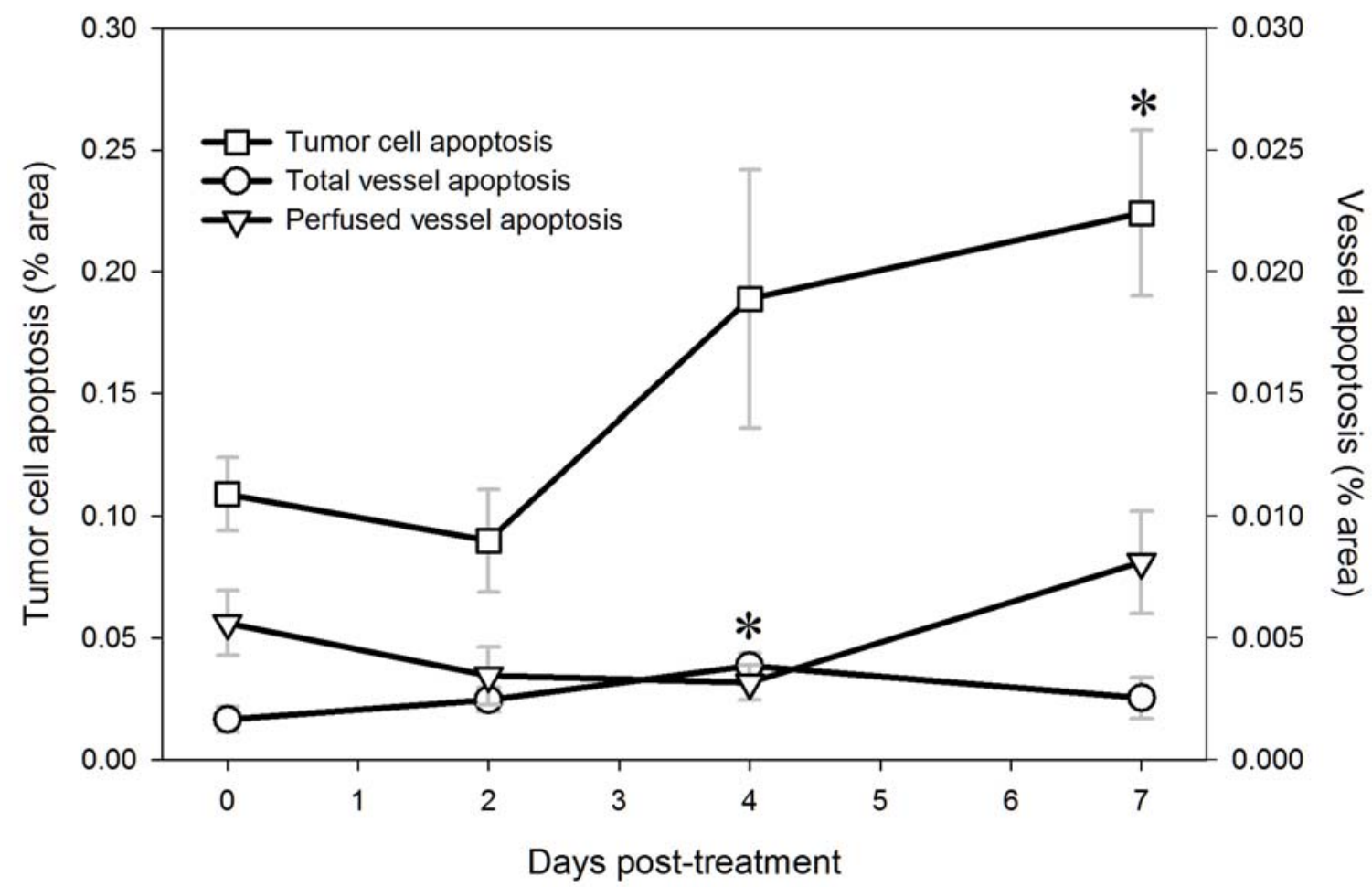

Figure 5 - Effect of combination AG-013736 + RT treatment on tumor cell and endothelial apoptosis in PC-3 tumors.

\section{Reportable Outcomes:}

Abstracts and Presentations:

1) 2005 Radiation Research Meeting, Denver, CO. Pathophysiological effects of antibodies to IGF-1R and VEGFR-2 plus fractionated radiation in DU145 prostate carcinoma xenografts. BM Fenton and SF Paoni.

2) 2005 University of Rochester 10th Annual Cancer Center Symposium, Rochester, NY. Pathophysiological effects of VEGF/PDGF receptor kinase inhibitors plus fractionated radiation in DU145 prostate carcinoma xenografts. BM Fenton and SF Paoni. 
3) 2005 International Society of Magnetic Resonance in Medicine Meeting, Miami, FL. Estimation of vascular functionality in two separate mammary carcinoma tumors during growth and exposure to carbogen using an interleaved DGE/DSE acquisition. PR Connelly, S Kennedy B Fenton, S Paoni, J Zhong.

4) 2006 American Association for Cancer Research Meeting, Washington, DC. Combined effects of VEGF/PDGF receptor tyrosine kinase inhibitors plus fractionated radiation on DU145 prostate carcinoma vasculature and oxygenation. BM Fenton and SF Paoni.

5) 2006 Tumor Microenvironment Meeting, Boston, MA. Antiangiogenic treatment enhances the tumor inhibitory effects of radiation. BM Fenton.

6) 2006 Society of Interventional Radiology Annual Meeting, San Diego, CA. Cone beam computer tomography angiogenesis imaging: preliminary studies. D Conover, R Ning, S Paoni, I Wilson, X Lu, Y Zhang, and B Fenton.

7) 2006 University of Rochester 11th Annual Cancer Center Symposium, Rochester, NY. Upstate NY Small Animal Imaging Resource Center cone beam computed tomography angiogenesis imaging preliminary studies. $D$ Conover, R Ning, S Paoni, I Wilson, X Lu, Y Zhang, B Fenton.

8) 2006 University of Rochester 11th Annual Cancer Center Symposium, Rochester, NY. Antiangiogenic strategies enhance the tumor inhibitory effects of radiation. BM Fenton.

9) 2007 13th International Congress of Radiation Research, San Francisco, CA. Combining antiangiogenic therapy with radiotherapy enhances tumor response without functionally normalizing the tumor vasculature. B Fenton and S Paoni.

10)2007 Innovative Minds in Prostate Cancer Today - IMPaCT Meeting, Atlanta, GA. Antivascular effects of VEGFR inhibition combined with radiotherapy in human DU145 prostate xenografts. B Fenton and S Paoni.

11)2007 Golden Horseshoe Meeting, Hamilton, Ontario, Canada. Evaluation of vascular 'normalization' via image analysis of multi-stained immunohistochemical sections. B Fenton.

\section{Publications:}

1) Fenton, BM and SF Paoni. The addition of AG-013736 to fractionated radiation improves tumor response without functionally normalizing the tumor vasculature. Cancer Res 67(20):9921-8, 2007.

2) D Conover, $R$ Ning, S Paoni, I Wilson, $X$ Lu, $Y$ Zhang, and B Fenton. Proceedings of SPIE 6143: 61433G1-6, Cone beam computer tomography angiogenesis imaging: preliminary studies, 2006.

Funding applied for based on this work:

1) $\mathrm{NIH} \mathrm{NCI} \mathrm{R01} \mathrm{-} \mathrm{Tumor} \mathrm{oxygenation,} \mathrm{vascularization,} \mathrm{and} \mathrm{radiation}$ response 
2) $\mathrm{NIH} \mathrm{NCl} \mathrm{R01} \mathrm{-} \mathrm{Monitoring} \mathrm{tumor} \mathrm{response} \mathrm{using} \mathrm{ultrasound} \mathrm{and}$ pathophysiological microimaging

Degrees obtained partially supported by this grant:

1) Patrick Connelly, PhD in Biomedical Engineering, University of Rochester, 2006

Personnel supported:

1) Bruce Fenton, $\mathrm{PhD}$

2) Scott Paoni, MS

3) P. Sabrina Agro, BS

\section{Conclusions:}

The current studies are the first to treat tumors with a combination of fractionated RT plus AG-013736, which is primarily a receptor tyrosine kinase inhibitor of VEGFRs at the dose levels used in this study. This combination resulted in a significantly reduced tumor growth rate in comparison to either monotherapy alone. For the combination treated tumors, tumor vascular density significantly decreased and hypoxia progressively increased compared to controls at weeks 1-3, but was not significantly different from either RT or AG-013736 at the week 2 timepoint. Functional vessel endothelial cell apoptosis was also increased by the combination. However, differences with respect to either monotherapy were minimal. Since alterations in total and perfused vessel spacings were generally proportionate, functional vasculature was most likely not selectively targeted by the combination therapy.

Previous work has suggested that VEGFR-2 inhibitors alone can lead to vascular normalization, which has been defined as a combination of selective pruning of excess tumor vessels, an increase in pericyte coverage, and a transient improvement in tumor oxygenation ${ }^{2,3}$. As summarized in recent reviews ${ }^{4,5}$, combinations of RT with antiangiogenic strategies have almost universally demonstrated increased tumor growth delay when compared to RT alone. Since neither irradiated nor unirradiated tumor cells are usually affected by antiangiogenic compounds in vitro ${ }^{5}$, this suggests a radiosensitizing effect that is either restricted to the endothelial cells, related to alterations in tumor blood flow, or both.

As a rule, pericytes are believed to form tighter associations with endothelial cells in normal tissue as compared to tumors ${ }^{6,7}$. However, the literature has been somewhat conflicted in defining optimal antibodies for identification of pericyte maturity. A major obstacle is the variability in marker expression and coverage among different tumor models $s^{6,8,9}$, although elegant studies have convincingly demonstrated that PDGFR $\beta+$ cells are a progenitor of $\alpha$-sma+ perivascular cells ${ }^{10}$ and that vessels covered by more mature $\alpha$-sma+ pericytes are selectively 
protected in tumors subjected to VEGF withdrawal ${ }^{8}$. Previous work has also suggested that pericyte coverage does not generally increase following RT alone $^{11}$, and can either increase ${ }^{3}$ or decrease ${ }^{10}$ following antiangiogenic strategies. Although pericytes can confer resistance to a variety of VEGFR targeting agents, the inclusion of specific PDFGR inhibitors has been shown to overcome this resistance, leading to vessel destabilization and regression ${ }^{12}$. Recent work suggests that the effects of AG-013736 are most likely predominantly due to VEGFR rather than PDGFR $\beta$ inhibition ${ }^{13}$. In spontaneous pancreatic tumors, AG-013736 was shown to produce a tightening of $\alpha$-sma+ pericyte coverage combined with a loss of both pericyte-coated and pericyte-free vessels ${ }^{7}$. Vascular area was found to decrease by $79 \%$, but $\alpha$-sma+ pericyte area was only reduced $33 \%$, suggesting a preferential targeting of non- $\alpha$-sma+ vessels. PDGFR $\beta$ + pericytes were unchanged following administration or withdrawal of AG-013736, and vessels that survived treatment were generally perfused.

This contrasts somewhat with current findings in which perfused and nonperfused vessels were nonselectively ablated by combination treatments. However, we did observe an initial increase in $\alpha$-sma+ coverage, combined with increased dissociation of both $\alpha$-sma+ and PDGFR $\beta+$ pericytes. This supports a selective ablation of non- $\alpha$-sma+ coated vessels, rather than a recruitment of $\alpha$ sma+ pericytes, since vascular counts also substantially decreased. This selectivity was lost by week 2 of treatment, however, and further vascular reductions were not mirrored by corresponding alterations in pericyte coverage. Instead, a significant dissociation of both $\alpha$-sma + and PDGFR $\beta+$ pericytes was observed following either AG-013736 or the combination.

Recent studies have also suggested that basement membrane sleeves are retained following endothelial destruction and can later provide a scaffolding for rapid vascular regrowth following cessation of VEGF inhibition ${ }^{13}$. We found a similar dissociation of type IV collagen vascular sleeves from endothelial cells as well as from PDGFR $\beta+$ pericytes following combination therapy. Interestingly, the timing of increases in PDGFR $\beta$ dissociation precisely paralleled the temporal reductions in vessel counts following both single and combined therapies. Together these data suggest that the net effect of the combination treatment is to selectively destroy endothelial cells, while at the same time stripping pericytes from their associated basement membranes.

In the current work, combination therapy was initially scheduled such that AG-013736 was delivered post-RT, under the assumption that pretreatment with the antiangiogenic agent would reduce tumor perfusion and thereby compromise $\mathrm{RT}^{14}$. Although later work demonstrated that combination treatment did, as predicted, reduce tumor perfusion and in some cases increase tumor hypoxia, the specific scheduling of radiation in relation to AG-013736 treatment had little ultimate effect on tumor response or pathophysiology. Although previous studies have reported increased endothelial and tumor cell apoptosis immediately 
following RT, alternative scheduling of the antiangiogenic agent either pre- or post-RT had no significant effect.

Since antiangiogenic strategies have only been shown effective when combined with conventional modalities such as RT or chemotherapy, increasing our understanding of the underlying mechanisms is a vital first step to optimizing treatment scheduling. Based on our work to date, we propose that studies seeking to quantify pathophysiological changes following combined modality treatments are most meaningful under two conditions: 1) a panel of pericyte markers is used to define treatment-induced changes in pericyte function, and 2) volume-matched controls rather than day-matched controls (or temporal changes during treatment) are used, which provide a more physiologically relevant comparison when evaluating treatment effects on tumor vascular function.

\section{References:}

1. Fenton BM and Paoni SF. The Addition of AG-013736 to Fractionated Radiation Improves Tumor Response without Functionally Normalizing the Tumor Vasculature. Cancer Res 2007;67:9921-8.

2. Tong RT, Boucher Y, Kozin SV, Winkler F, Hicklin DJ, Jain RK. Vascular normalization by vascular endothelial growth factor receptor 2 blockade induces a pressure gradient across the vasculature and improves drug penetration in tumors. Cancer Res 2004;64:3731-6.

3. Winkler F, Kozin SV, Tong RT, et al. Kinetics of vascular normalization by VEGFR2 blockade governs brain tumor response to radiation; Role of oxygenation, angiopoietin-1, and matrix metalloproteinases. Cancer Cell 2004;6:553-63.

4. Kobayashi $\mathrm{H}$ and Lin PC. Antiangiogenic and radiotherapy for cancer treatment. Histol Histopathol 2006;21:1125-34.

5. Nieder C, Wiedenmann N, Andratschke N, Molls M. Current status of angiogenesis inhibitors combined with radiation therapy. Cancer Treat Rev 2006;32:348-3.

6. Morikawa S, Baluk P, Kaidoh T, Haskell A, Jain RK, McDonald DM. Abnormalities in pericytes on blood vessels and endothelial sprouts in tumors. Am J Pathol 2002;160:985-1000.

7. Inai $\mathrm{T}$, Mancuso $\mathrm{M}$, Hashizume $\mathrm{H}$, et al. Inhibition of vascular endothelial growth factor (VEGF) signaling in cancer causes loss of endothelial fenestrations, regression of tumor vessels, and appearance of basement membrane ghosts. Am J Pathol 2004;165:35-52.

8. Benjamin LE, Golijanin D, Itin A, Pode D, Keshet E. Selective ablation of immature blood vessels in established human tumors follows vascular endothelial growth factor withdrawal. Journal of Clinical Investigation 1999;103:159-65.

9. Eberhard A, Kahlert S, Goede V, Hemmerlein B, Plate KH, Augustin HG. Heterogeneity of angiogenesis and blood vessel maturation in human 
tumors: Implications for antiangiogenic tumor therapies. Cancer Res 2000;60:1388-93.

10. Song S, Ewald AJ, Stallcup W, Werb Z, Bergers G. PDGFRbeta+ perivascular progenitor cells in tumours regulate pericyte differentiation and vascular survival. Nat Cell Biol 2005;7:870-9.

11. Tsai JH, Makonnen S, Feldman M, Sehgal CM, Maity A, Lee WM. lonizing Radiation Inhibits Tumor Neovascularization by Inducing Ineffective Angiogenesis. Cancer Biol Ther 2005;4:1395-400.

12. Bergers G, Song S, Meyer-Morse N, Bergsland E, Hanahan D. Benefits of targeting both pericytes and endothelial cells in the tumor vasculature with kinase inhibitors. J Clin Invest 2003;111:1287-95.

13. Mancuso MR, Davis R, Norberg SM, et al. Rapid vascular regrowth in tumors after reversal of VEGF inhibition. J Clin Invest 2006;116:2610-21.

14. Williams KJ, Telfer BA, Brave S, et al. ZD6474, a potent inhibitor of vascular endothelial growth factor signaling, combined with radiotherapy: scheduledependent enhancement of antitumor activity. Clin Cancer Res 2004;10:8587-93.

\section{Appendices:}

Manuscripts:

1) Fenton, BM and SF Paoni. The addition of AG-013736 to fractionated radiation improves tumor response without functionally normalizing the tumor vasculature. Cancer Res 67(20):9921-8, 2007. 


\title{
The Addition of AG-013736 to Fractionated Radiation Improves Tumor Response without Functionally Normalizing the Tumor Vasculature
}

\author{
Bruce M. Fenton and Scott F. Paoni \\ Department of Radiation Oncology, University of Rochester Medical Center, Rochester, New York
}

\begin{abstract}
Although antiangiogenic strategies have proven highly promising in preclinical studies and some recent clinical trials, generally only combinations with cytotoxic therapies have shown clinical effectiveness. An ongoing question has been whether conventional therapies are enhanced or compromised by antiangiogenic agents. The present studies were designed to determine the pathophysiologic consequences of both single and combined treatments using fractionated radiotherapy plus AG-013736, a receptor tyrosine kinase inhibitor that preferentially inhibits vascular endothelial growth factor receptors. DU145 human prostate xenograft tumors were treated with (a) vehicle alone, (b) AG-013736, (c) $5 \times 2 \mathrm{~Gy} /$ wk radiotherapy fractions, or $(d)$ the combination. Automated image processing of immunohistochemical images was used to determine total and perfused blood vessel spacing, overall hypoxia, pericyte/collagen coverage, proliferation, and apoptosis. Combination therapy produced an increased tumor response compared with either monotherapy alone. Vascular density progressively declined in concert with slightly increased $\alpha$-smooth muscle actin-positive pericyte coverage and increased overall tumor hypoxia (compared with controls). Although functional vessel endothelial apoptosis was selectively increased, reductions in total and perfused vessels were generally proportionate, suggesting that functional vasculature was not specifically targeted by combination therapy. These results argue against either an AG-013736- or a combination treatment-induced functional normalization of the tumor vasculature. Vascular ablation was mirrored by the increased appearance of dissociated pericytes and empty type IV collagen sleeves. Despite the progressive decrease in tumor oxygenation over 3 weeks of treatment, combination therapy remained effective and tumor progression was minimal. [Cancer Res 2007;67(20):9921-8]
\end{abstract}

\section{Introduction}

Although antiangiogenic strategies have been shown highly promising in preclinical studies and some recent phase II and III clinical trials (1), generally only combinations of antiangiogenic and cytotoxic therapies have shown clinical effectiveness. As recently reviewed (2), an ongoing question about such combined therapies has been whether conventional therapies, such as radiotherapy or chemotherapy, are enhanced or compromised by anti-

Requests for reprints: Bruce M. Fenton, Department of Radiation Oncology, University of Rochester Medical Center, Box 704, 601 Elmwood Avenue, Rochester, NY 14642. Phone: 585-275-7911; Fax: 585-273-1042; E-mail: bruce.fenton@rochester.edu.

(C)2007 American Association for Cancer Research.

doi:10.1158/0008-5472.CAN-07-1066 angiogenic agents. Although antiangiogenic strategies might be expected to destroy tumor vasculature and thereby deprive the tumor of radiosensitizing oxygen or hinder access to chemotherapeutic agents, results are inconclusive. Some workers have found decreased tumor oxygenation and blood flow following such agents $(3,4)$, whereas others have shown the opposite $(5,6)$. Concurrent (4), postradiotherapy $(7,8)$, and preradiotherapy $(9,10)$ combination scheduling with antiangiogenics have all been shown advantageous (11).

An intriguing hypothesis recently reviewed by Jain (12) contends that vascular endothelial growth factor (VEGF) inhibitors can selectively prune the chaotic and inefficient vasculature commonly found in tumors, resulting in a transiently normalized vascular configuration. This improved vasculature, characterized by reduced vessel counts and increased coverage of periendothelial support cells, or pericytes, is thus predicted to more efficiently deliver both oxygen and drugs. A second often quoted hypothesis is that the susceptibility of established tumor blood vessels to interference with VEGF/VEGF receptor-2 (VEGFR-2) signaling may be limited to vessels that lack pericyte coverage (13-15). Recent studies, however, have noted that pericytes can also be actively recruited before pruning (6).

The present investigation was designed to evaluate the consequences of combining fractionated radiation with AG-013736, a potent receptor tyrosine kinase inhibitor of VEGFRs that also inhibits platelet-derived growth factor receptors (PDGFR) at higher doses. Does vascular density decrease following 1 to 3 weeks of single or combination treatment, and if so, does tumor hypoxia increase or instead decrease in line with vascular normalization? Given the well-known dependence of radiosensitivity on tumor oxygen levels, any increase in hypoxia would presumably also compromise further combination therapy. Our second objective was to compare alterations in the tightness and coverage of two pericyte markers and to determine the relation between pericyte coverage and selective vascular ablation. PDGFR $\beta$ was selected as a marker of perivascular progenitors or less mature pericytes (16), whereas $\alpha$-smooth muscle actin ( $\alpha$-sma) was chosen to distinguish more mature pericytes or vascular smooth muscle cells $(16,17)$.

The current findings argue against a functional normalization of the tumor vasculature following combination therapy. Although treatment reduced vascular densities, overall tumor hypoxia progressively increased. Despite this decrease in oxygenation, however, combination therapy remained effective and tumor growth was inhibited.

\section{Materials and Methods}

Tumor and animal models. The DU145 human prostate carcinoma cell line was obtained from the American Type Culture Collection and maintained in DMEM (Mediatech-Cellgro) supplemented with $10 \%$ fetal bovine serum. Viable tumor cells $\left(10^{7}\right)$ were implanted into the left hind legs 
of $\mathrm{NCr} n u / n u$ male mice and grown to tumor volumes of 200 to $400 \mathrm{~mm}^{3}$. Tumor (including leg) diameters were measured thrice weekly using a graduated hole template, with opposite nontumor leg diameters subtracted to calculate actual tumor volumes: tumor volume $=\pi / 6 \times$ (tumor leg diameter $^{3}-$ nontumor leg diameter ${ }^{3}$ ). Mice were housed in microisolator cages and given food and water ad libitum. Guidelines for the humane treatment of animals were followed as approved by the University Committee on Animal Resources.

Treatments. AG-013736, a receptor kinase inhibitor of VEGFRs and, at higher doses, PDGFRs $\left(\mathrm{IC}_{50}=0.1 \mathrm{nmol} / \mathrm{L}\right.$ for VEGFR-1, $0.2 \mathrm{nmol} / \mathrm{L}$ for VEGFR-2, 0.1-0.3 nmol/L for VEGFR-3, and $1.6 \mathrm{nmol} / \mathrm{L}$ for PDGFRß; ref. 18), was provided by Pfizer Global Research and given once daily by gavage in a volume of $0.13 \mathrm{~mL}$. Control animals received $0.5 \%$ carboxymethylcellulose drug carrier. Irradiations were done on nonanesthetized mice using a ${ }^{137} \mathrm{Cs}$ source operating at $2.4 \mathrm{~Gy} / \mathrm{min}$. Mice were confined to plastic jigs with tumor-bearing legs extended through an opening in the side, allowing local irradiations. Fractionated doses were given in five daily 2 Gy fractions per week (omitting weekends). For combination treatments, radiotherapy was delivered first, and AG-013736 was given within $\sim 4 \mathrm{~h}$. Mice were sacrificed, and tumors were excised and then quick frozen (using liquid nitrogen) following 1, 2, or 3 weeks of treatment.

DiOC $_{7}$ perfusion marker and EF5 hypoxia marker. To visualize blood vessels open to flow, $\mathrm{DiOC}_{7}$, an intravascular stain that preferentially stains cells immediately adjacent to the vessels, was injected intravascularly by tail vein $1 \mathrm{~min}$ before freezing (19). Localized areas of tumor hypoxia were assessed in 9.0- $\mu \mathrm{m}$ frozen sections (one section taken as near as possible to the tumor center for each combination of stains) by immunohistochemical identification of sites of 2-nitroimidazole metabolism (20). A pentafluorinated derivative of etanidazole (EF5) was injected intravascularly $(0.2 \mathrm{~mL}$ of $10 \mathrm{mmol} / \mathrm{L} \mathrm{EF5}$ ) $1 \mathrm{~h}$ before tumor freezing (21) followed by a second dose 45 min later. Regions of high EF5 metabolism were visualized using a Cy3 fluorochrome (Amersham) conjugated to the ELK3-51 antibody, which is extremely specific for the EF5 adducts that form when the drug is incorporated by hypoxic cells (22). Both the EF5 (made by the National Cancer Institute) and the ELK3-51 were obtained from the University of Pennsylvania Imaging Service Center (C. Koch, Director).

Immunohistochemistry and image acquisition. Tumor sections were imaged using a $10 \times$ objective (Olympus BX40 microscope), digitized (QImaging Retiga 1300C Peltier-cooled, 12-bit digital camera), background corrected, and image analyzed using Image-Pro software (Media Cybernetics). Twelve-bit gray-scale image montages from 16 adjacent microscope fields (encompassing a total area of $21.6 \mathrm{~mm}^{2}$ ) were automatically acquired and digitally combined for multiple stains (20). First, images of the $\mathrm{DiOC}_{7}$ were obtained immediately after cryosectioning and fixing in cold acetone for $10 \mathrm{~min}$. Following staining, this section was returned to identical stage coordinates to obtain images of both EF5/Cy3 and an endothelial cell marker [MECA-32 (biotinylated primary, 1:100; PharMingen) followed by incubation with Vectastain ABC Elite Standard kit and AEC detection (Vector Laboratories)].

Adjacent frozen sections were sliced to visualize combinations of antibodies, generally dual staining of endothelial cells (MECA-32) versus markers for either (a) proliferation [Ki-67 (MIB-1 clone, mouse anti-human, 1:150; Dako) in conjunction with the M.O.M. "mouse primary antibody on mouse tissue" kit (Vector Laboratories)], (b) apoptosis [FITC-terminal deoxynucleotidyl transferase-mediated dUTP nick end labeling (TUNEL) assay, DeadEnd Fluorometric TUNEL System (Promega), with the following modifications: sections were fixed in $4 \%$ neutral buffered formalin for 10 min after panendothelial cell antigen staining, washed in PBS, and then incubated for 15 min with $0.2 \%$ Triton X-100 before apoptosis staining], $(c)$ pericytes [PDGFR $\beta$, clone APB5, rat anti-mouse, 1:50 (eBioscience) or $\alpha$-sma, clone 1A4, mouse Cy3 directly conjugated, 1:1,000 (Sigma)], or $(d)$ vascular basement membrane (type IV collagen, polyclonal rabbit antimouse, 1:2,000; Chemicon). For Ki-67 and apoptosis, 9- $\mu \mathrm{m}$ sections were fixed in cold acetone for $10 \mathrm{~min}$, air dried for $10 \mathrm{~min}$, and stored at $-80^{\circ} \mathrm{C}$. For pericytes, $20-\mu \mathrm{m}$ sections were fixed for $10 \mathrm{~s}$ in $1 \%$ neutral buffered formalin, washed and covered in PBS, imaged for $\mathrm{DiOC}_{7}, \mathrm{PBS}$ aspirated, fixed in room temperature acetone for $1 \mathrm{~min}$, and air dried for $30 \mathrm{~min}$ before staining. For type IV collagen, 20- $\mu \mathrm{m}$ sections were fixed in warm acetone for $1 \mathrm{~min}$ and air dried for $30 \mathrm{~min}$. Secondary antibody detection was done using species-specific Alexa Fluor 488 (type IV collagen) or 546 (Ki-67, PDGFR $\beta$; 1:500; Molecular Probes) combined with MECA-32 using contrasting Alexa Fluor secondary antibodies. Sections were coverslipped with Slow Fade Gold antifading agent (Molecular Probes).

Image analysis: vascular spacing, apoptosis, and proliferation. As described previously (23), tumor blood vessel spacing was determined using a combination of image segmentation and distance map filtering to obtain a spatial sampling of distance filter intensities, which are directly proportional to the distribution of distances to the nearest vessel. These distances (dependent on tumor blood vessel spacing) are reflective of the median distances over which oxygen and nutrients must diffuse to reach all cells of the tumor.

Colocalized and thresholded images of MECA-32 endothelial cell staining, $\mathrm{DiOC}_{7}$, TUNEL, and Ki-67 staining were obtained, and percentage area was determined for each as well as for the overlap between TUNEL and perfused vessel staining (using custom Image-Pro macros). Percentage apoptotic vessel overlap was calculated by dividing the area of vessel apoptosis overlap by the perfused or total vessel area.

Image analysis: collagen and pericyte coverage and tightness. Colocalized and thresholded stained images of MECA-32, $\alpha$-sma, and PDGFR $\beta$ were used to obtain percentage areas and overlap between endothelial and pericyte markers. Percentage coverage was defined as the percentage area overlap divided by the percentage area endothelial cells, and percentage dissociation was the percentage pericyte area that did not overlap endothelial cells. Percentage areas of PDGFR $\beta^{+}$cells, endothelial cells, and type IV collagen staining were determined using the Image-Pro percentage area measurement in combination with thresholded images of positive staining.

Statistical analysis. Treatments were compared using Student's $t$ test or the Mann-Whitney rank sum test and considered significant for $P \leq 0.05$.

\section{Results}

Effects of AG-013736 and/or fractionated radiotherapy on tumor growth. Mice bearing DU145 tumors were first treated with 10,25 , or $50 \mathrm{mg} / \mathrm{kg} / \mathrm{d}$ AG-013736 to define a dose that would slow but not stop tumor growth, thus allowing the later effects of combination therapies to be recognized. Tumor volumes were measured thrice weekly, mice were sorted into groups having roughly equal mean volumes, and treatments were initiated at volumes between 200 and $400 \mathrm{~mm}^{3}$. Figure $1 A$ presents percentage tumor volume increase as a function of AG-013736 dose. Tumor responses following doses of 10 and $25 \mathrm{mg} / \mathrm{kg}$ (by gavage) were roughly equivalent, but $50 \mathrm{mg} / \mathrm{kg}$ resulted in substantial inhibition of tumor growth over the 3 weeks of treatment. A dose of $25 \mathrm{mg} / \mathrm{kg}$ was therefore selected for the combination experiments. Based on pharmacokinetic data, once daily dosing of AG-013736 at $25 \mathrm{mg} / \mathrm{kg}$ only provided transient (2-4 h per day) inhibition of PDGFR $\beta$ (data not shown). Preliminary studies also showed that a schedule of $5 \times 2 \mathrm{~Gy} / \mathrm{wk}$ of radiation slowed tumor growth to a rate similar to that following $25 \mathrm{mg} / \mathrm{kg}$ AG-013736 (Fig. $1 B$ ).

Mice were next treated for a period of 2 weeks with either fractionated radiation $(5 \times 2 \mathrm{~Gy} / \mathrm{wk})$ or AG-013736 $(25 \mathrm{mg} / \mathrm{kg} / \mathrm{d})$ and 1 to 3 weeks for the combination. As shown in Fig. $1 B$, tumor volume at the end of 2 weeks was significantly reduced for either single or combination treatments. Percentage increases in tumor volume were similar between radiotherapy $(40 \pm 9.8 \%)$ and AG$013736(48 \pm 9.2 \%)$, and the combination was markedly reduced versus controls $(12 \pm 5.7 \%$ versus $77 \pm 11 \% ; P<0.001)$. A separate experiment measured combination and control volumes over 3 weeks of treatment, at which time the percentage increases in tumor volume were $23.3 \pm 14 \%$ and $211 \pm 57 \%$, respectively $(P<0.001)$. 
Figure 1. Percentage increase in tumor volume as a function of days following initiation of treatment. $A$, growth delay in response to three different doses of AG-013736 [vehicle $(O), 10 \mathrm{mg} / \mathrm{kg} / \mathrm{d}(\Delta)$, $25 \mathrm{mg} / \mathrm{kg} / \mathrm{d}(\boldsymbol{\Delta})$, and $50 \mathrm{mg} / \mathrm{kg} / \mathrm{d}(\bullet)]$. ,+ AG-013736 treatment days. Mean tumor volumes at treatment initiation ranged from 220 to $300 \mathrm{~mm}^{3}$, and five to six tumors were included per group. $B$, growth delay in response to vehicle $(O)$, fractionated radiotherapy $(R T ; 5 \times 2 \mathrm{~Gy} / \mathrm{wk} ; \Delta)$, $25 \mathrm{mg} / \mathrm{kg} \mathrm{AG}-013736(\boldsymbol{\Delta})$, or radiotherapy + AG-013736 (•). +, treatment days for AG-013736; $x$, treatment days for 2 Gy radiotherapy fractions. Points, mean tumor volumes at treatment initiation ranged from 360 to $490 \mathrm{~mm}^{3}$ and seven to nine tumors were included per group; bars, SE.
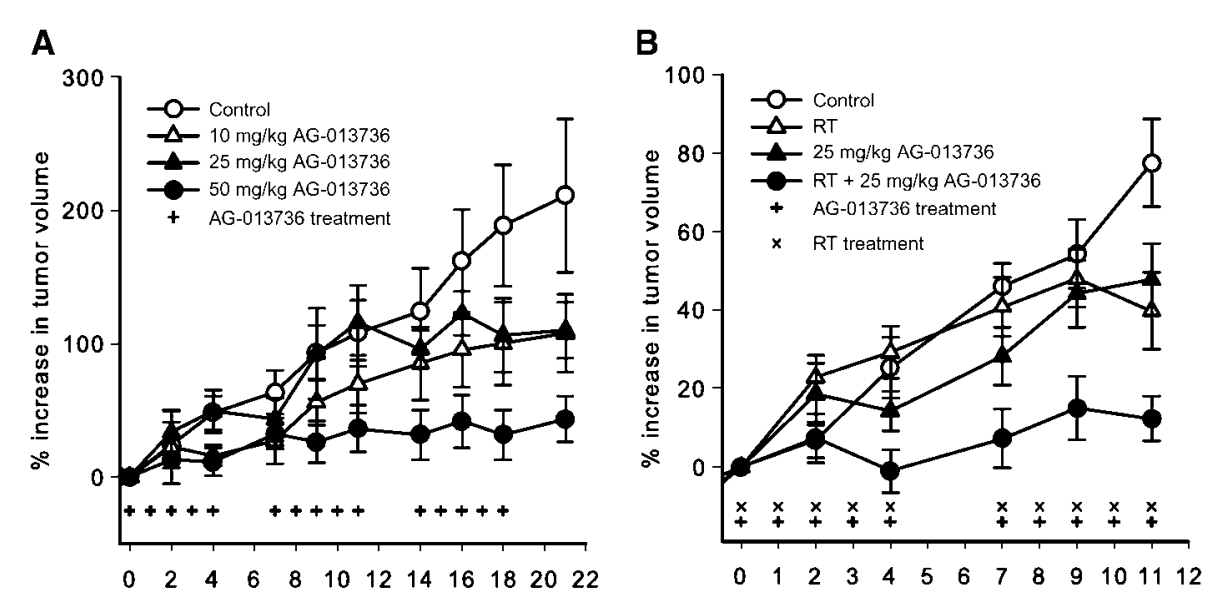

Days post treatment initiation
Total and perfused blood vessel spacing increases following either single or combination treatments. We next examined the effects of treatment on total and perfused vascular spacing to gauge whether radiotherapy, AG-013736, or the combination affected tumor vascular spacing. As shown in Fig. $2 A, 2$ weeks of AG-013736, radiotherapy, or the combination significantly increased both total (black columns) and perfused (white columns) blood vessel spacing, although none of the three treatments was significantly different from each other. In this and the following figures, treated tumors could have been compared with either day-matched or volume-matched controls. To minimize pathophysiologic variations due to tumor growth alone, our usual approach is to favor the volume-matched comparisons. Thus, week 1 controls $\left(\right.$ mean volumes $=560 \pm 30 \mathrm{~mm}^{3}$ ) were chosen for comparison
Figure 2. Effect of treatment on tumor vascular spacing and hypoxia. $A$, effect of 2 wk of treatment on total (black columns) and perfused (white columns) vessel spacing (10 d of treatments) compared with week 1 controls. AG, AG-013736. $B$, effect of 1 to $3 \mathrm{wk}$ of combination treatment on total and perfused vessel spacing $(5,10$, or 15 combination treatments of 2 Gy radiotherapy and then

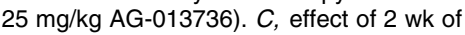
treatment on mean overall tumor hypoxia. $D$, effect of 1 to $3 \mathrm{wk}$ of combination treatment on mean overall tumor hypoxia (five to eight tumors per group). Columns, mean; bars, SE. *, significant differences of treated tumors in relation to week 1 controls $(P \leq 0.05)$.

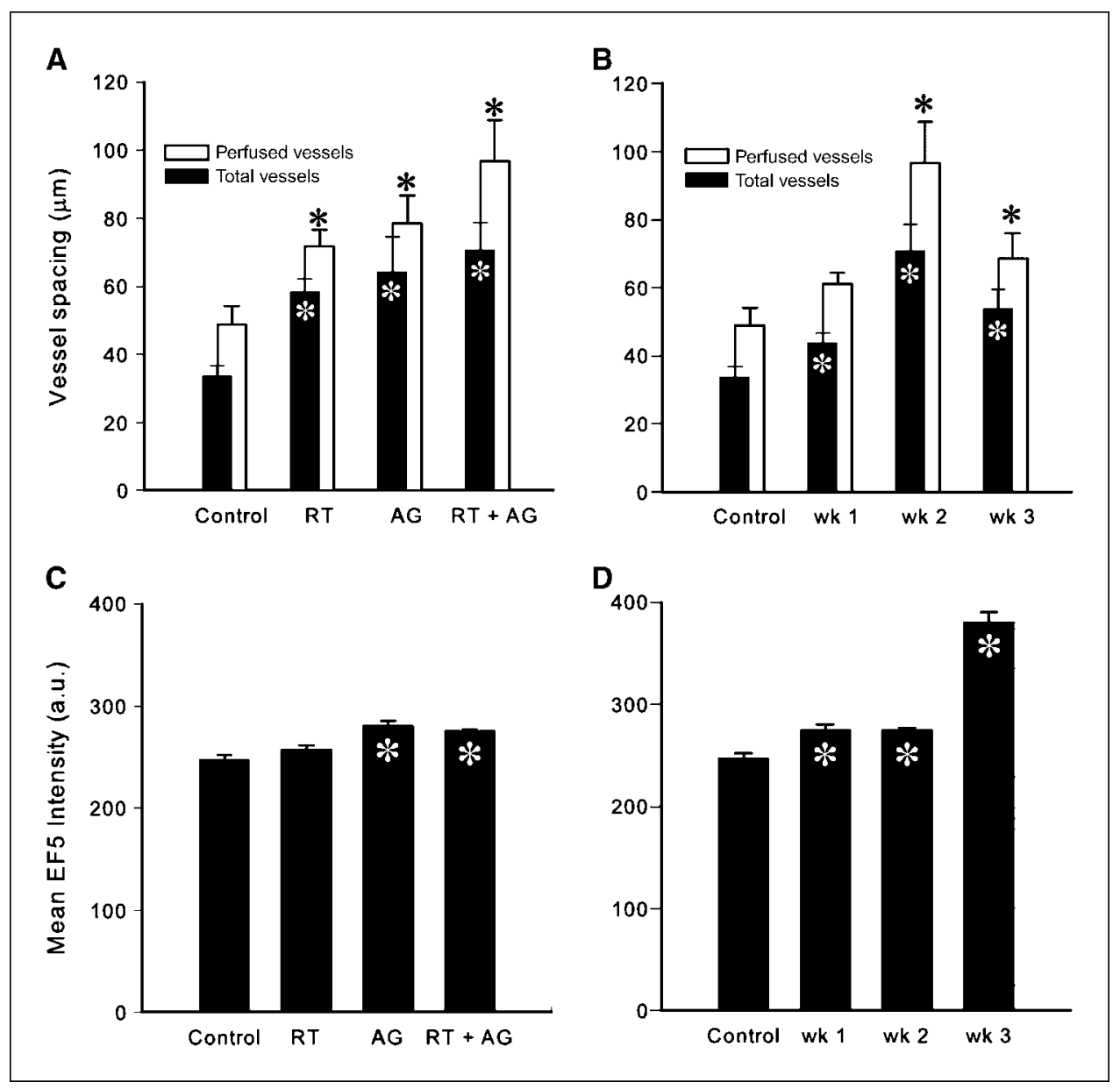


because they were a fairly close match to all of the week 2 treated tumor volumes (which ranged from $350 \pm 30 \mathrm{~mm}^{3}$ for the combination-treated tumors to $530 \pm 60$ for the AG-013736). It should be noted, however, that in the case of this relatively slowgrowing DU145 tumor model, vessel spacing and hypoxia did not change significantly for the control tumors over the range from 560 to $850 \mathrm{~mm}^{3}$. Figure $2 B$ compares week 1 controls to combination-treated tumors frozen at 1,2 , or 3 weeks. Although the combination produced significant increases in total and perfused vessel spacing at most time points in relation to week 1 controls, changes were minimal at week 1 , most pronounced at week 2, and less pronounced by week 3 (which were not significantly different from week 2 for either total or perfused).

Tumor hypoxia increases following combination therapy. Overall tumor hypoxia was quantified in frozen sections based on the intensity of a fluorescently conjugated antibody to EF5. Perfused spacing increased substantially at week 2 for all three treatments (Fig. 2A), but hypoxia was unchanged for the radiotherapy $(P=0.29)$ and increased only minimally for either AG-013736 $(P=0.011)$ or the combination $(P=0.033)$, which were no different from each other $(P=0.35)$. Overall hypoxia increased slightly at weeks 1 and 2 in combination-treated tumors $(P=0.004$ and 0.002 , respectively; Fig. $2 D$ ) and substantially at week 3 $(P<0.001)$. Thus, although tumor volumes remained essentially constant following combination therapy, vascular functionality steadily decreased with an ultimate increase in tumor hypoxia.
Although alterations in hypoxia might be expected to parallel changes in perfused vessel spacing, this was not always the case. For instance, perfused vessel spacing increased substantially between weeks 1 and 2 of treatment, whereas overall tumor hypoxia remained constant. At week 3, perfused spacing was not significantly different from weeks 1 or $2(P=0.063)$, but hypoxia markedly increased $(P=0.003)$. As has been shown previously using direct measures of tumor microvessel $\mathrm{HbO}_{2}$ saturations (24), vessels defined as "perfused" can vary markedly in terms of their functionality. Thus, the constant levels of perfused vessel spacing over the 3 weeks of treatment may correspond to a progressive decrease in functionality with continued treatment, leading to the observed increase in hypoxia. We therefore consider the EF5 intensities as the more rigorous assay for changes in overall tumor hypoxia following treatment. This index is incapable of distinguishing between clonogenic and nonclonogenic tumor cell subpopulations, however, which can only be definitively defined using survival or clonogenic assays of "radiobiological hypoxic fraction" (25).

Combination therapy increases perfused vessel endothelial apoptosis and reduces tumor cell proliferation. Based on TUNEL staining, neither overall tumor cell apoptotic density nor endothelial cell apoptosis was significantly increased by either single or combination treatments (data not shown). Apoptosis of perfused vessel endothelial cells (Fig. $3 A$ and $B$ ) increased significantly for combination-treated tumors at weeks 2 and 3 $(P=0.03$ versus 0.097 for radiotherapy alone and 0.201 for

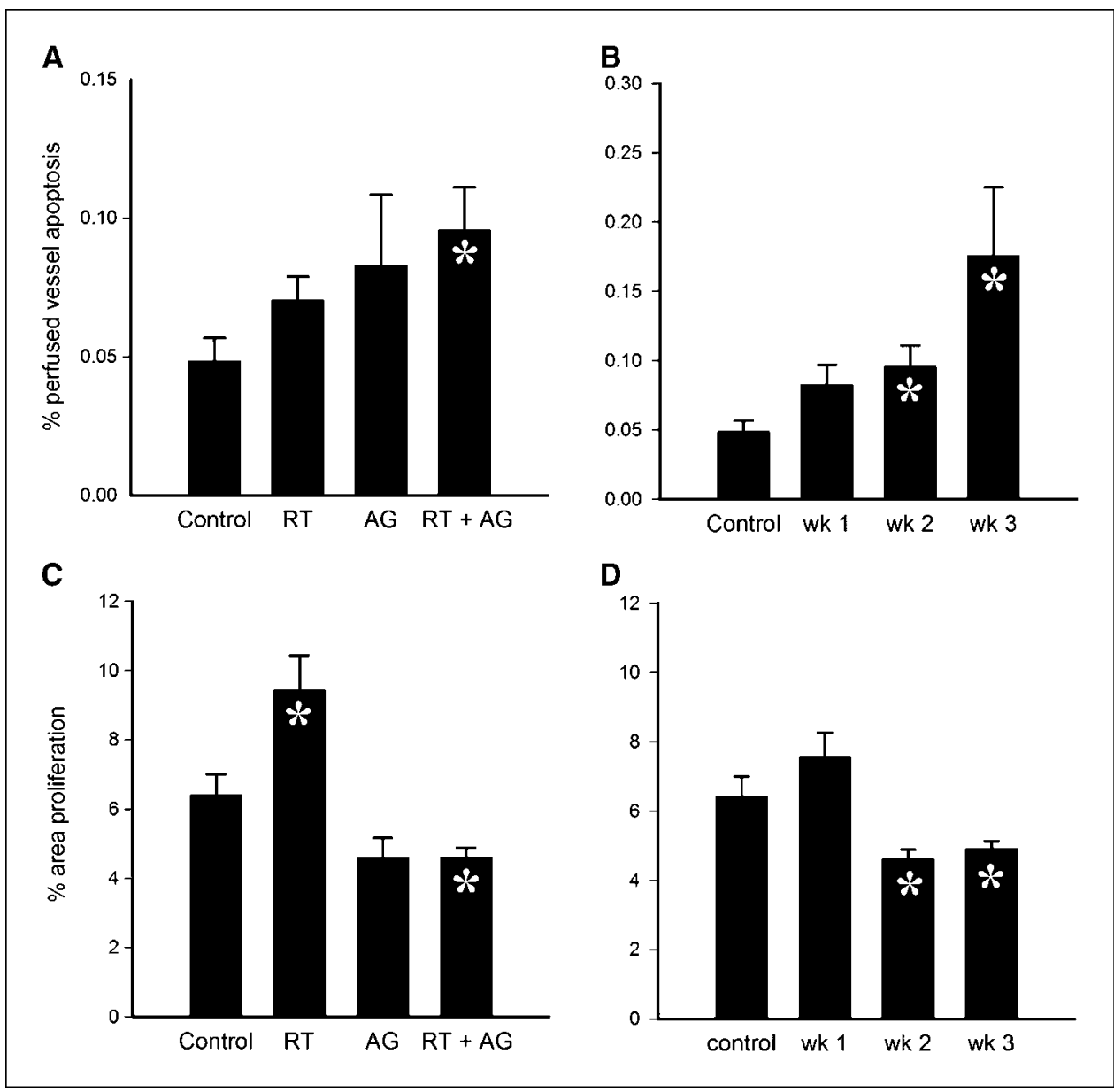

Figure 3. Effects of treatment on perfused vessel endothelial apoptosis and \% area tumor cell proliferation. $A$, effect of 2 wk of treatment (2 Gy radiotherapy plus $25 \mathrm{mg} / \mathrm{kg}$ AG-013736 per day) on \% perfused vessel apoptosis, defined as the area of overlap between perfused vessel staining $\left(\mathrm{DiOC}_{7}\right)$ and TUNEL staining for apoptosis divided by the total perfused vessel staining. $B$, effect of 1 to $3 \mathrm{wk}$ of combination treatment on $\%$ perfused vessel apoptosis. $C$, effect of 2 wk of treatments on $\%$ area proliferation (defined as the ratio of $\mathrm{Ki}-67^{+}$-stained area divided by total tumor cell area). $D$, effect of 1 to $3 \mathrm{wk}$ of combination treatment on $\%$ area proliferation. Columns, mean; bars, SE.

*, significant differences of treated tumors in relation to week 1 controls $(P \leq 0.05)$. 
AG-013736 alone). This could reflect increased drug and oxygen concentrations within the vessels, assuming that these vessels were also perfused at time of treatment. No significant differences were noted between combination treatments and either radiotherapy or AG-013736 alone ( $P=0.22$ or 0.66 , respectively). Finally, based on percentage positive Ki-67 staining, tumor cell proliferation was significantly increased by radiotherapy at 2 weeks after therapy (Fig. $3 C$ ) but decreased by the combination at both 2 and 3 weeks (Fig. $3 D$ ). Again, the combination was not significantly different from AG-013736 alone.

PDGFR $\beta^{+}$pericyte dissociation, but not coverage, is increased by combination treatment. $P D G F R \beta^{+}$is generally believed to mark a progenitor population of pericytes, whereas $\alpha$-sma ${ }^{+}$denotes more mature cells (16). Figure 4 presents effects of treatment on $(a)$ percentage PDGFR $\beta^{+}$coverage (i.e., the fraction of blood vessel area covered by PDGFR $\beta^{+}$pericytes) and $(b)$ percentage dissociation (the fraction of PDGFR $\beta^{+}$pericyte area not overlapping blood vessels). In the current study, vascular coverage of PDGFR $\beta^{+}$pericytes was $\sim 75 \%$ in controls and was not significantly altered by single or combined treatments (Fig. $4 A$ and $B$, yellow columns). Dissociation of PDGFR $\beta^{+}$pericytes, however, increased progressively for radiotherapy, AG-013736, and the combination (Fig. 4C) and remained elevated throughout the 3 weeks of combination therapy (Fig. $4 D$ ). Dissociation in the combination tumors was significantly higher than for the AG-013736 $(P=0.014)$, which in turn was significantly higher than for the radiotherapy $(P=0.038)$. Interestingly, dissociation in response to the combination peaked at week 2 , which corresponds to the peak in vascular spacing (Fig. $2 B$ ) and suggests a possible link between vascular ablation and pericyte dissociation. Representative pseudocolor images of tumors dual stained for endothelial cells and both PDGFR $^{+}$and $\alpha$-sma ${ }^{+}$pericytes are shown in Fig. $4 E$.

$\alpha$-sma ${ }^{+}$pericyte dissociation and coverage are increased by combination treatment. Coverage of $\alpha$-sma ${ }^{+}$pericytes was substantially lower than for PDGFR $\beta^{+}$pericytes in untreated tumor vessels $(\sim 20 \%$ versus $\sim 75 \%)$ but increased following both radiotherapy or the combination and remained elevated throughout 3 weeks of therapy (Fig. $4 A$ and $B$, blue columns). This suggests either $(a)$ selective ablation of non- $\alpha$-sma ${ }^{+}$pericyte-coated blood vessels or $(b)$ recruitment of $\alpha$-sma ${ }^{+}$pericytes to previously noncoated vessels. Because vessel numbers were also substantially decreased at these times, selective ablation seems the more likely mechanism after week 1 of combination therapy. At weeks 2 to 3 , however, vessel counts were further reduced in the absence of increased $\alpha$-sma ${ }^{+}$coverage (Fig. $4 B$, blue columns), suggesting that vessels are then similarly targeted irrespective of pericyte coverage. As with PDGF $\beta^{+}$cells, dissociation of $\alpha$-sma ${ }^{+}$cells increased significantly with both mono and combined therapies and progressively increased with increasing weeks of combination therapy (Fig. $4 C$ and D, blue columns).

Type IV collagen-coated basement membranes are retained following endothelial ablation. Figure 5 illustrates changes in percentage areas of endothelial cells, PDGFR $\beta^{+}$pericytes, $\alpha$-sma ${ }^{+}$ pericytes, and type IV collagen (used to identify vascular basement membranes) following combination treatment. As expected based on the increased vascular spacing results of Fig. $2 B$, percentage area of endothelial cells decreased significantly in relation to controls at each time point $(P<0.001$ in each case). In contrast, no significant changes were seen for type IV collagen, $\alpha$-sma, or PDGFR $\beta$ percentage areas. This suggests that the net effect of the combination therapy was to ablate the endothelial cell layer of the blood vessels while retaining the basement membrane sleeve and perivascular cells, as has been previously reported following AG013736 alone (26). In control tumors, dual staining revealed extensive overlap between type IV collagen ${ }^{+}$basement membrane and endothelial cell staining (Fig. 5B, yellow), although small subregions with high proportions of disassociated collagen sleeves were also sometimes present. For combination-treated tumors, in contrast, vascular counts were substantially reduced and empty collagen sleeves were broadly distributed throughout the tumor cross-section (Fig. $5 \mathrm{C}$ ). Finally, overlap between PDGFR $\beta^{+}$and collagen was also substantially reduced following combination therapy (compare Fig. $5 D$ and $E$ ), suggesting that treatment results in the dissociation of basement membrane sleeves from pericytes as well as endothelial cells.

\section{Discussion}

The current studies are the first to treat tumors with a combination of fractionated radiotherapy plus AG-013736, a receptor tyrosine kinase inhibitor of primarily VEGFRs at the dose levels used in this study. This combination resulted in a significantly reduced tumor growth rate in comparison with either monotherapy alone. For the combination-treated tumors, tumor vascular density significantly decreased and hypoxia progressively increased compared with controls but was not significantly different from either radiotherapy or AG-013736 at the week 2 time point. Functional vessel endothelial cell apoptosis was also increased by the combination. However, differences with respect to either monotherapy were again minimal. Because alterations in total and perfused vessel spacings were generally proportionate, functional vasculature was likely not selectively targeted by the combination therapy.

Previous work has suggested that VEGFR-2 inhibitors alone can lead to vascular normalization, which has been defined as a combination of selective pruning of excess tumor vessels, an increase in pericyte coverage, and a transient improvement in tumor oxygenation $(6,27)$. In agreement with the current results, however, alternate studies have shown short-term and long-term impairment of vascular function and increased tumor hypoxia following VEGFR-2 inhibition (3). As summarized in recent reviews $(11,28)$, combinations of radiotherapy with antiangiogenic strategies have almost universally shown increased tumor growth delay when compared with radiotherapy alone. Because neither irradiated nor unirradiated tumor cells are usually affected by antiangiogenic compounds in vitro (11), this suggests a radiosensitizing effect that is either restricted to the endothelial cells, related to alterations in tumor blood flow, or both. Endothelial sensitization is supported by the work of Schueneman et al. (29), who administered daily radiotherapy after SU11248, a similar multitargeted small-molecule inhibitor of VEGFRs and PDGFRs, and found that the combination reduced tumor blood flow but increased endothelial-specific apoptosis and vascular destruction. SU11657, another inhibitor of VEGFRs and PDGFRs, was most effective when single-dose radiotherapy was delivered 1 day after the drug. At that time, tumor interstitial pressures were substantially reduced and tumor blood flow was presumably increased (9).

As a rule, pericytes are believed to form tighter associations with endothelial cells in normal tissue compared with tumors $(17,30)$. However, the literature has been somewhat conflicted in defining optimal antibodies for identification of pericyte maturity. A major obstacle is the variability in marker expression and coverage among different tumor models $(13,17,31)$, although elegant studies 
have convincingly shown that $\mathrm{PDGFR}^{+}$cells are a progenitor of $\alpha$-sma ${ }^{+}$perivascular cells (16) and that vessels covered by more mature $\alpha$-sma ${ }^{+}$pericytes are selectively protected in tumors subjected to VEGF withdrawal (13). Previous work has also suggested that pericyte coverage does not generally increase following radiotherapy alone (32) and can either increase (6) or decrease (16) following antiangiogenic strategies. Although pericytes can confer resistance to a variety of VEGFR-targeting agents, the inclusion of specific PDFGR inhibitors has been shown to overcome this resistance, leading to vessel destabilization and regression (15).
Recent work suggests that the effects of AG-013736 are most likely predominantly due to VEGFR rather than PDGFR $\beta$ inhibition (26). In spontaneous pancreatic tumors, AG-013736 was shown to produce a tightening of $\alpha$-sma ${ }^{+}$pericyte coverage combined with a loss of both pericyte-coated and pericyte-free vessels (30). Vascular area was found to decrease by $79 \%$, but $\alpha$-sma ${ }^{+}$pericyte area was only reduced $33 \%$, suggesting a preferential targeting of non- $\alpha$-sma ${ }^{+}$ vessels. PDGFR $\beta^{+}$pericytes were unchanged following administration or withdrawal of AG-013736, and vessels that survived treatment were generally perfused.

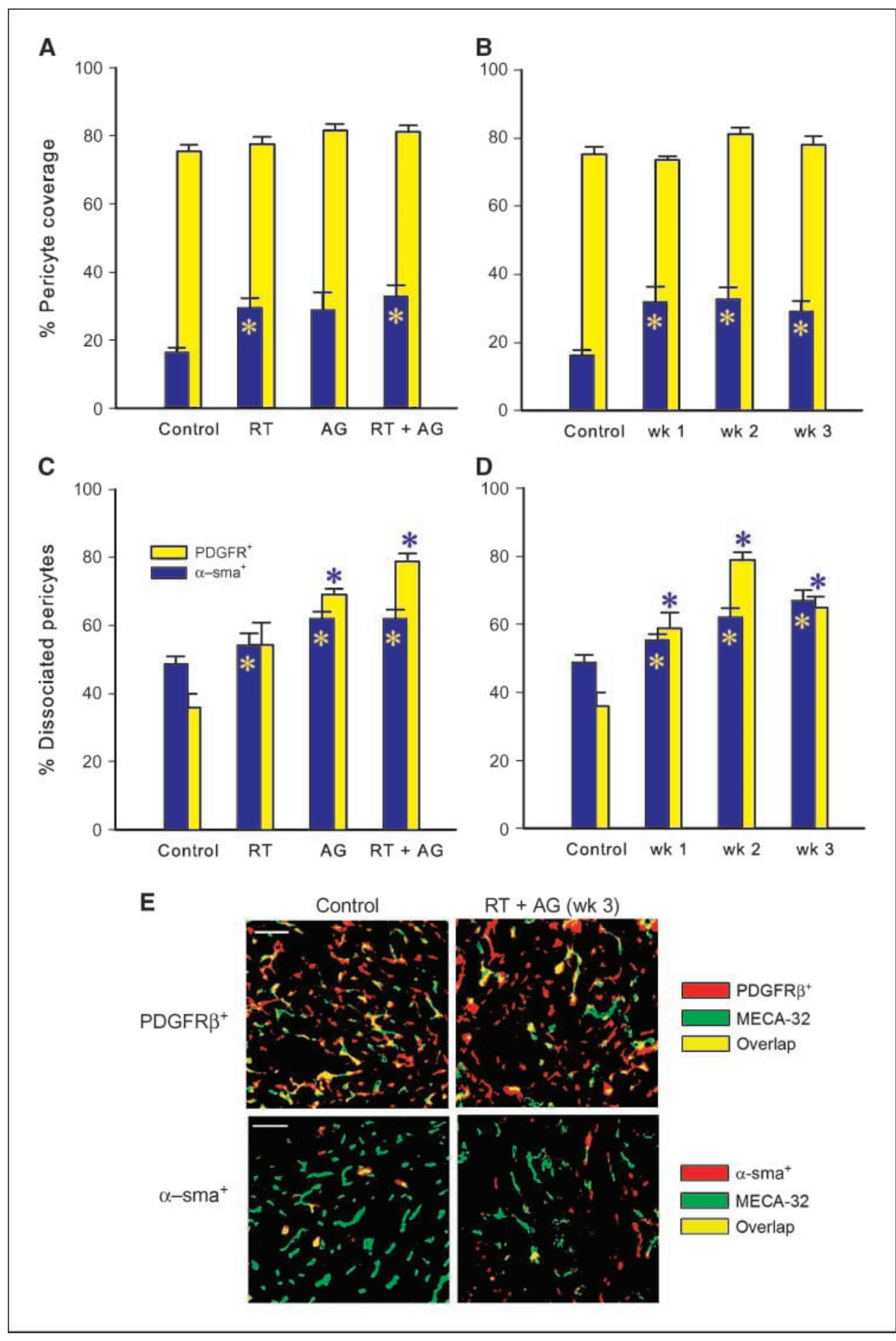

Figure 4. Effect of treatment on pericyte $\%$ coverage and dissociation. $A$, effect of 2 wk of treatment ( 2 Gy radiotherapy plus $25 \mathrm{mg} / \mathrm{kg} \mathrm{AG-013736} \mathrm{per} \mathrm{day)} \mathrm{on} \mathrm{\%}$ coverage (defined as the ratio of area overlap between endothelial cells and pericytes divided by the total area of endothelial cells) for PDGFR $\beta^{+}$(yellow columns) and $\alpha$-sma ${ }^{+}$(blue columns). $B$, effect of 1 to 3 wk of combination treatment on $\%$ coverage. $C$, effect of $2 \mathrm{wk}$ of treatment on \% dissociated pericytes (defined as the ratio of the area of pericytes not overlapping with endothelial cells divided by the total pericyte area). Columns, mean; bars, SE. *, significant differences of treated tumors in relation to week 1 controls $(P \leq 0.05)$. $D$, effect of 1 to 3 wk of combination treatment on \% dissociated pericytes. $E$, representative thresholded, pseudocolor images of $\alpha$-sma ${ }^{+}($red $)$or PDGFR $\beta^{+}$pericytes (red), MECA-32 endothelial marker (green), and overlap (yellow). Bar, $100 \mu \mathrm{m}$. 


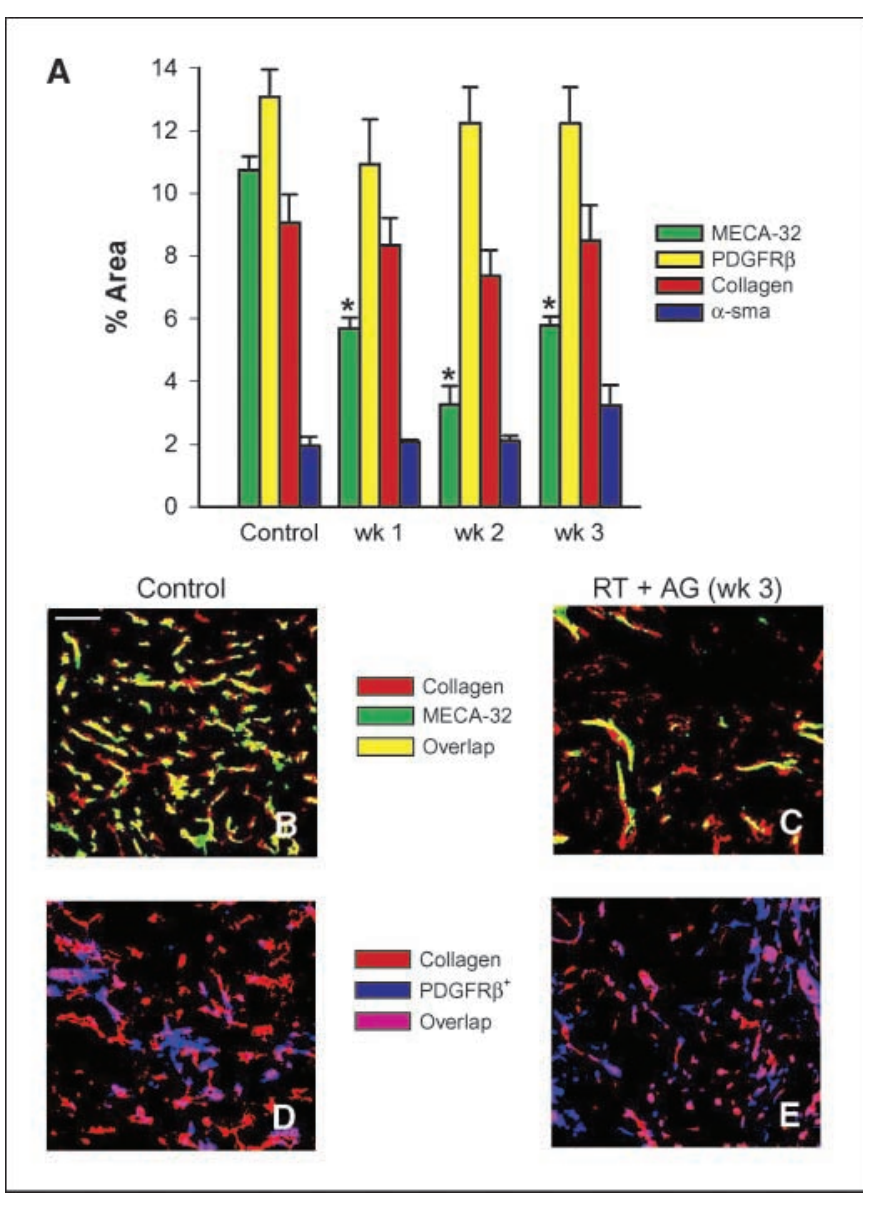

Figure 5. $A$, comparison of the effects of 1 to $3 \mathrm{wk}$ of combination treatment ( 2 Gy radiotherapy plus $25 \mathrm{mg} / \mathrm{kg} \mathrm{AG-013736} \mathrm{per} \mathrm{day)} \mathrm{on} \mathrm{\%} \mathrm{area} \mathrm{of} \mathrm{total}$ vessels (MECA-32), pericytes (PDGFR $\beta^{+}$and $\alpha-$ sma $^{+}$), and vascular basement membranes (type IV collagen). Only total vessel \% area changed significantly with treatment. Columns, mean; bars, SE. *, significant differences of treated tumors compared with week 1 controls $(P<0.001$ for each). $B$ and $C$, pseudocolored, dual-stained images of type IV collagen (red), MECA-32 endothelial marker (green), and overlap (yellow). Bar, $100 \mu \mathrm{m}$. B, week 1 contro tumor showing predominant overlap (yellow) between type IV collagen (red) and endothelial cells (green). $C$, week 3 combination-treated tumor showing reduced vascular counts and a high proportion of basement membrane sleeves. $D$ and $E$, pseudocolored, dual-stained images of type IV collagen (red), PDGFR $^{+}$(blue), and overlap (magenta). D, week 1 control tumor showing extensive overlap (magenta) between collagen (red) and PDGFR $\beta^{+}$(blue). $E$, week 3 combination-treated tumor showing increase in dissociation of PDGFR $\beta^{+}$cells from type IV collagen $(r e d)$.

This contrasts somewhat with current findings in which perfused and nonperfused vessels were nonselectively ablated by combination treatments. However, we did observe an initial increase in $\alpha$-sma ${ }^{+}$coverage, combined with increased dissociation of both $\alpha$-sma ${ }^{+}$and PDGFR $\beta^{+}$pericytes. This supports a selective ablation of non- $\alpha$-sma ${ }^{+}$-coated vessels rather than a recruitment of $\alpha$-sma ${ }^{+}$pericytes because vascular counts also substantially decreased. This selectivity was lost by week 2 of treatment, however, and further vascular reductions were not mirrored by corresponding alterations in pericyte coverage. Instead, a significant dissociation of both $\alpha$-sma ${ }^{+}$and PDGFR $\beta^{+}$pericytes was observed following either AG-013736 or the combination.

Recent studies have also suggested that basement membrane sleeves are retained following endothelial destruction and can later provide a scaffolding for rapid vascular regrowth following cessation of VEGF inhibition (26). We found a similar dissociation of type IV collagen vascular sleeves from endothelial cells as well as from PDGFR $\beta^{+}$pericytes following combination therapy. Interestingly, the timing of increases in PDGFR $\beta$ dissociation precisely paralleled the temporal reductions in vessel counts following both single and combined therapies. Together, these data suggest that the net effect of the combination treatment is to selectively destroy endothelial cells while at the same time stripping pericytes from their associated basement membranes.

In the current experimental design, combination therapy was scheduled such that AG-013736 was delivered after radiotherapy under the assumption that pretreatment with the antiangiogenic agent would reduce tumor perfusion and thereby compromise radiotherapy (8). AG-013736 could alternatively have been administered just before radiotherapy, however, which would presumably further accentuate endothelial apoptosis and sensitization of endothelial cells to radiotherapy. Although previous studies have reported increased endothelial and tumor cell apoptosis immediately following radiotherapy, both return to baseline levels by 1 and 2 weeks (32). Our weekly time points could conceivably have missed transient changes, which illustrates the importance of including multiple time points.

In conclusion, the current findings argue against a treatmentinduced functional normalization of the tumor vasculature when applying combination therapy. Rather than tightening pericytes, AG-013736 and the combination treatment served instead to loosen pericyte-vessel and pericyte-basement membrane associations in this tumor model. Treatment substantially reduced total and functional vascular densities, but overall tumor hypoxia progressively increased, in contrast to the normalization hypothesis. Despite the reduction in oxygenation, tumor progression was minimal over 3 weeks of combination treatment, most likely due to continued vascular destruction and the prevention of new vessel growth. Further studies are essential to extend these measurements to additional tumor models and to determine whether alternative scheduling may also enhance treatment response.

\section{Acknowledgments}

Received 3/21/2007; revised 7/16/2007; accepted 7/27/2007.

Grant support: Department of Defense grant W81XWH-04-1-0827 and National Cancer Institute grant CA52586.

The costs of publication of this article were defrayed in part by the payment of page charges. This article must therefore be hereby marked advertisement in accordance with 18 U.S.C. Section 1734 solely to indicate this fact.

We thank Drs. David Shalinsky and Dana Hu-Lowe (Pfizer Global Research, La Jolla CA) for providing the AG-013736 small-molecule inhibitor and P. Sabrina Agro for technical assistance.

\section{References}

1. George DJ. Phase 2 studies of sunitinib and AG013736 in patients with cytokine-refractory renal cell carcinoma. Clin Cancer Res 2007;13:753-7s.

2. Horsman MR, Siemann DW. Pathophysiological effects of vascular-targeting agents and the implications for combination with conventional therapies. Cancer Res 2006;66:11520-39.

3. Franco M, Man S, Chen L, et al. Targeted anti-vascular endothelial growth factor receptor-2 therapy leads to short-term and long-term impairment of vascular function and increase in tumor hypoxia. Cancer Res 2006;66:3639-48.

4. Riesterer O, Honer M, Jochum W, Oehler C, Ametamey S, Pruschy M. Ionizing radiation antagonizes tumor hypoxia induced by antiangiogenic treatment. Clin Cancer Res 2006;12:3518-24. 
5. Fenton BM, Paoni SF, Grimwood BG, Ding I. Disparate effects of endostatin on tumor vascular perfusion and hypoxia in two murine mammary carcinomas. Int J Radiat Oncol Biol Phys 2003;57:1038-46.

6. Winkler F, Kozin SV, Tong RT, et al. Kinetics of vascular normalization by VEGFR2 blockade governs brain tumor response to radiation: role of oxygenation, angiopoietin-1, and matrix metalloproteinases. Cancer Cell 2004:6:553-63.

7. Zips D, Krause M, Hessel F, et al. Experimental study on different combination schedules of VEGF-receptor inhibitor PTK787/ZK222584 and fractionated irradiation. Anticancer Res 2003;23:3869-76.

8. Williams KJ, Telfer BA, Brave S, et al. ZD6474, a potent inhibitor of vascular endothelial growth factor signaling, combined with radiotherapy: schedule-dependent enhancement of antitumor activity. Clin Cancer Res 2004;10:8587-93.

9. Huber PE, Bischof M, Jenne J, et al. Trimodal cancer treatment: beneficial effects of combined antiangiogenesis, radiation, and chemotherapy. Cancer Res 2005; 65:3643-55.

10. Cao C, Albert JM, Geng L, et al. Vascular endothelial growth factor tyrosine kinase inhibitor AZD2171 and fractionated radiotherapy in mouse models of lung cancer. Cancer Res 2006;66:11409-15.

11. Nieder C, Wiedenmann N, Andratschke N, Molls M Current status of angiogenesis inhibitors combined with radiation therapy. Cancer Treat Rev 2006;32:348-64.

12. Jain RK. Normalization of tumor vasculature: an emerging concept in antiangiogenic therapy. Science 2005;307:58-62.

13. Benjamin LE, Golijanin D, Itin A, Pode D, Keshet E. Selective ablation of immature blood vessels in established human tumors follows vascular endothelia growth factor withdrawal. J Clin Invest 1999;103:159-65. 14. Benjamin LE, Hemo I, Keshet E. A plasticity window for blood vessel remodelling is defined by pericyte coverage of the preformed endothelial network and is regulated by PDGF-B and VEGF. Development 1998;125: 1591-8.

15. Bergers G, Song S, Meyer-Morse N, Bergsland E, Hanahan D. Benefits of targeting both pericytes and endothelial cells in the tumor vasculature with kinase inhibitors. J Clin Invest 2003;111:1287-95.

16. Song S, Ewald AJ, Stallcup W, Werb Z, Bergers G. PDGFR $^{+}$perivascular progenitor cells in tumours regulate pericyte differentiation and vascular survival. Nat Cell Biol 2005;7:870-9.

17. Morikawa S, Baluk P, Kaidoh T, Haskell A, Jain RK, McDonald DM. Abnormalities in pericytes on blood vessels and endothelial sprouts in tumors. Am J Pathol 2002;160:985-1000.

18. Wickman G, Hallin M, Dillon $R$, et al. Further characterization of the potent VEGF/PDGF receptor tyrosine kinase inhibitor, AG-013736 in preclinical tumor models for its antiangiogenesis and antitumor activity. Proc Am Assoc Cancer Res 2003;44:A3780.

19. Trotter MJ, Chaplin DJ, Olive PL. Use of a carbocyanine dye as a marker of functional vasculature in murine tumours. Br J Cancer 1989;59:706-9.

20. Fenton BM, Paoni SF, Lee J, Koch CJ, Lord EM. Quantification of tumor vascular development and hypoxia by immunohistochemical staining and $\mathrm{HbO}_{2}$ saturation measurements. Br J Cancer 1999;79:464-71. 21. Fenton BM, Lord EM, Paoni SF. Effects of radiation on tumor intravascular oxygenation, vascular configuration, hypoxic development, and survival. Radiat Res 2001;155:360-8.

22. Lord EM, Harwell L, Koch CJ. Detection of hypoxic cells by monoclonal antibody recognizing 2-nitroimidazole adducts. Cancer Res 1993;53:5721-6.

23. Fenton BM, Paoni SF, Ding I. Effect of VEGF receptor-2 antibody on vascular function and oxygenation in spontaneous and transplanted tumors. Radiother Oncol 2004;72:221-30.
24. Fenton BM, Rofstad EK, Degner FL, Sutherland RM. Cryospectrophotometric determination of tumor intravascular oxyhemoglobin saturations: dependence on vascular geometry and tumor growth. J Natl Cancer Inst 1988;80:1612-9.

25. Fenton BM, Kiani MF, Siemann DW. Should direct measurements of tumor oxygenation relate to the radiobiological hypoxic fraction of a tumor? Int J Radiat Oncol Biol Phys 1995;33:365-73.

26. Mancuso MR, Davis R, Norberg SM, et al. Rapid vascular regrowth in tumors after reversal of VEGF inhibition. J Clin Invest 2006;116:2610-21.

27. Tong RT, Boucher Y, Kozin SV, Winkler F, Hicklin DJ, Jain RK. Vascular normalization by vascular endothelial growth factor receptor 2 blockade induces a pressure gradient across the vasculature and improves drug penetration in tumors. Cancer Res 2004;64:3731-6.

28. Kobayashi H, Lin PC. Antiangiogenic and radiotherapy for cancer treatment. Histol Histopathol 2006;21: $1125-34$.

29. Schueneman AJ, Himmelfarb E, Geng L, et al SU11248 maintenance therapy prevents tumor regrowth after fractionated irradiation of murine tumor models. Cancer Res 2003;63:4009-16.

30. Inai $T$, Mancuso $M$, Hashizume $H$, et al. Inhibition of vascular endothelial growth factor (VEGF) signaling in cancer causes loss of endothelial fenestrations, regression of tumor vessels, and appearance of basement membrane ghosts. Am J Pathol 2004;165:35-52.

31. Eberhard A, Kahlert S, Goede V, Hemmerlein B, Plate KH, Augustin HG. Heterogeneity of angiogenesis and blood vessel maturation in human tumors: implications for antiangiogenic tumor therapies. Cancer Res 2000;60: 1388-93.

32. Tsai JH, Makonnen S, Feldman M, Sehgal CM, Maity A, Lee WM. Ionizing radiation inhibits tumor neovascularization by inducing ineffective angiogenesis. Cancer Biol Ther 2005;4:1395-400. 